\title{
Pathogenicity, immunogenicity, and protective ability of an attenuated SARS-CoV-2 variant with a deletion at the $\mathrm{S} 1 / \mathrm{S} 2$ junction of the spike protein
}

\section{Pui Wang}

University of Hong Kong

\section{Siu-Ying Lau}

The University of Hong Kong

\section{Shaofeng Deng}

The University of Hong Kong

\section{Pin Chen}

University of Hong Kong

\section{Bobo Mok}

The University of Hong Kong

Jinxia Zhang

Department of microbiology

\section{Andrew Lee}

University of Hong Kong

\section{Kwok-Hung Chan}

The University of Hong Kong

\section{Rachel Tam}

University of Hong Kong

\section{Wenjun Song}

The University of Hong Kong https://orcid.org/0000-0002-0165-7894

\section{Kelvin To}

The University of Hong Kong https://orcid.org/0000-0002-1921-5824

\section{Jasper Chan}

The University of Hong Kong

\section{Kwok-Yung Yuen}

University of Hong Kong https://orcid.org/0000-0002-2083-1552

Honglin Chen ( $\nabla$ hlchen@hku.hk)

University of Hong Kong https://orcid.org/0000-0001-5108-8338 
Keywords: COVID-19, SARS-CoV-2, vaccine development, S1/S2 junction, pathogenicity, immunogenicity, protective ability, Ca-DelMut

Posted Date: September 24th, 2020

DOI: https://doi.org/10.21203/rs.3.rs-78635/v1

License: (c) (1) This work is licensed under a Creative Commons Attribution 4.0 International License. Read Full License

Version of Record: A version of this preprint was published at Nature Communications on May 13th, 2021. See the published version at https://doi.org/10.1038/s41467-021-23166-0. 
1 Pathogenicity, immunogenicity, and protective ability of an attenuated SARS-CoV-2 variant with a deletion at the S1/S2 junction of the spike protein

3

4 Pui Wang ${ }^{1}$, Siu-Ying Lau ${ }^{1}$, Shaofeng Deng ${ }^{1}$, Pin Chen ${ }^{1}$, Bobo Wing-Yee Mok ${ }^{1}$, Anna Jinxia

5 Zhang ${ }^{1}$, Andrew Chak-Yiu Lee ${ }^{1}$, Kwok-Hung Chan ${ }^{1}$, Rachel Chun-Yee Tam ${ }^{1}$,Wenjun Song

$6{ }^{1,2}$, Kelvin Kai-Wang To ${ }^{1}$, Jasper Fuk-Woo Chan ${ }^{1}$, Kwok-Yung Yuen ${ }^{1}$ and Honglin Chen $^{1 *}$

$9 \quad{ }^{1}$ Department of Microbiology and State Key Laboratory for Emerging Infectious Diseases, Li

Ka Shing Faculty of Medicine, The University of Hong Kong, Hong Kong SAR,

2. State Key Laboratory of Respiratory Disease, Institute of Integration of Traditional and Western Medicine, The First Affiliated Hospital of Guangzhou Medical University, Guangzhou Medical University, 195 Dong Feng Road (W), Guangzhou 510180, China

*Correspondence to: Honglin Chen, Department of Microbiology and State Key Laboratory for Emerging Infectious Diseases, Li Ka Shing Faculty of Medicine, The University of Hong Kong, Hong Kong SAR, China (hlchen@hku.hk) 


\section{Abstract}

27 SARS-CoV-2 is zoonotic origin and contains a PRRA polybasic cleavage motif which is 28 considered critical for efficient infection and transmission in humans. We previously reported 29 on a panel of attenuated SARS-CoV-2 variants with deletion at the S1/S2 junction of spike 30 protein. Here we characterize pathogenicity, immunogenicity, and protective ability of a further 31 cell-adapted SARS-CoV-2 variant, Ca-DelMut, in in vitro and in vivo systems. Ca-DelMut 32 replicates more efficiently than wild type or parental virus in cells, but causes no apparent 33 disease in hamsters, despite replicating in respiratory tissues. Unlike wild type virus, Ca34 DelMut causes no apparent pathological changes and does not induce elevated 35 proinflammatory cytokines in hamster infections, but still triggers a strong neutralizing 36 antibody response in hamsters. Ca-DelMut immunized hamsters challenged with wild type

37 SARS-CoV-2 are fully protected with no sign of virus replication in the upper or lower 38 respiratory tract of challenged animals, demonstrating sterilizing immunity. 
40 The emergence of SARS-CoV-2, a novel zoonotic origin $\beta$-coronavirus, has led to the

41 first documented pandemic caused by a coronavirus ${ }^{1,2}$. The virus continues to circulate

42 globally in humans with rapidly increasing numbers of infections and casualties each day

43 (https://coronavirus. jhu.edu/map.html). While coronaviruses from bats and pangolins have

44 been found to be closely related to SARS-CoV-2 ${ }^{3-6}$, the direct ancestral virus which attained

45 cross-species transmission and the intermediate animal host source of human infections have

46 not been defined. In the past two decades, three coronaviruses have jumped the species barrier

47 to infect humans ${ }^{7}$. SARS-CoV and MERS-CoV show limited human to human transmission

48 ability while causing severe disease and mortality. In contrast, SARS-CoV-2, which uses the

49 same human ACE2 binding receptor as SARS-CoV ${ }^{6}$, is highly transmissible and causes

50 variable severity of disease, from asymptomatic infections to severe and fatal outcomes.

51 Analysis of the SARS-CoV-2 genome reveals a distinct PRRA polybasic cleavage motif at the

$52 \mathrm{~S} 1 / \mathrm{S} 2$ junction of the spike protein when compared to the most proximal animal coronaviruses

53 yet detected ${ }^{8}$. Cleavage of spike into S1 and S2 subunits by the cellular protease furin

54 promotes cell fusion mediated by S2, which is critical for cellular entry of coronavirus ${ }^{9}{ }^{10}$. In

55 avian influenza virus, acquisition of a polybasic cleavage motif facilitates infection of an

56 increased variety of cell types ${ }^{11-13}$. This polybasic cleavage site is essential for infection of

57 human lung cells ${ }^{14}$. It is speculated that acquisition of the PRRA polybasic motif in the spike

58 protein of SARS-CoV-2 provides the virus with its unique ability of cross species transmission;

59 this motif therefore appears to serve as a pathogenic element in human infections ${ }^{10,14}$. If the

60 PRRA polybasic cleavage motif was not a natural functional component in the original virus

61 and acquired after cross species transmission, it is postulated that it may not be stable during

62 the infection of its new hosts, or may need further adaptation. Indeed, a panel of SARS-CoV-

632 variants with various lengths of deletion spanning the PRRA polybasic cleavage motif at the

64 spike protein $\mathrm{S} 1 / \mathrm{S} 2$ junction were identified in cultured cells and at low levels in clinical 
65 specimens, suggesting the S1/S2 junction may be under selection pressure as the SARS-CoV-

662 virus circulates in humans ${ }^{15,16}$. Initial characterization revealed that deletion at the S1/S2

67 junction causes SARS-CoV-2 virus attenuation in hamsters and prompted a further study to

68 understand the properties of these deletion mutants in in vitro and in vivo systems. It remains

69 to be determined whether some of these deletion variants may become more prevalent in

70 humans as their circulation continues. On the other hand, these attenuated SARS-CoV-2

71 variants may hold promise as candidate live vaccines and it is important that their potential in

72 this regard be evaluated.

73 To understand the role of the polybasic cleavage site in the infection and replication of

74 SARS-CoV-2 and determine the stability of deletion variants, a previously characterized

75 deletion mutant ${ }^{15}$, Del-Mut-1, was further adapted in Vero cells to obtain a highly attenuated

76 variant of SARS-CoV-2 virus, designated Ca-DelMut. We analyzed the growth properties of

77 this variant in cells and evaluated its pathogenicity in a hamster model. While it is attenuated

78 in the ability to cause disease in animals, Ca-DelMut replicates to a higher titer in Vero cells

79 than the wild type SARS-CoV-2 virus. High titers of neutralizing antibodies were detected in

80 animals previously infected with Ca-DelMut variant. However, infection with Ca-DelMut does

81 not induce the high levels of proinflammatory cytokines seen in wild type virus infections.

82 Importantly, Ca-DelMut immunized hamsters showed full protection with sterilizing immunity

83 against challenge with two different strains of wild type virus. 


\section{Results}

\section{Growth and pathogenic properties of Ca-DelMut in vitro and in vivo}

We previously identified and characterized a panel of SARS-CoV-2 variants containing $15-30 \mathrm{bp}$ deletions at the S1/S2 junction of the spike protein. One of the variants, Del-Mut-1, which contains a 30bp deletion spanningthe PRRA polybasic cleavage motif was shown to be attenuated in a hamster infection model ${ }^{15}$. We further passaged this mutant in Vero E6 cells and obtained a variant with additional mutations in multiple genes in the background of DelMut-1, designated Ca-DelMut (Figure 1 and Supplementary Table 1). Growth properties of Ca-DelMut, parental Del-Mut-1 and a wild type (HK-13) SARS-CoV-2 were analyzed in Vero E6 and Calu-3 cells. It is interesting to note that in Vero E6 cells both Del-Mut-1 and CaDelMut grow to a significantly higher titer at the 24 and 48 -hour time points than the wild type virus, with Ca-DelMut showing the strongest growth ability in both cell types (Figure 2A).

Notably, Ca-DelMut exhibits a cold adaptation phenotype in vitro, as demonstrated by comparatively high level of replication at $30^{\circ} \mathrm{C}$, whereas wild type virus (HK-13) replicates poorly at this temperature (Figure S1). Attenuation of Del-Mut-1 was reported previously ${ }^{15}$. To further characterize the Ca-DelMut variant of SARS-CoV-2, we infected hamsters with variant and wild type SARS-CoV-2 strains. While inoculation with $10^{3}$ pfu HK-13 caused significant body weight loss post-infection, no apparent body weight loss was observed in CaDelMut $\left(1.25 \times 10^{5}\right.$ and $\left.10^{3} \mathrm{pfu}\right)$ infected hamsters (Figure 2B). Histopathological analysis showed only mild regional alveolar septal infiltration and blood vessel congestion, with no obvious bronchiolar epithelium desquamation or luminal debris, no alveolar space infiltration or exudation, and no pathological changes in the intestines of Ca-DelMut infected hamsters, which are markedly different from the severe pathology observed in wild type virus infected animals (Figure S2). Examination of virus replication in lung and nasal turbinate tissues showed that Ca-DelMut replicates actively in the nasal turbinate tissues but that replication 
efficiency is much lower than that of HK-13 in hamster lungs (Figure 2C). These results indicate that $\mathrm{Ca}$-DelMut has altered tissue tropism and is more likely to infect and replicate in the upper respiratory tract.

\section{Immune response to infection with Ca-DelMut in hamsters}

Impaired or dysfunctional immune responses have been characterized as an important mechanism of pathogenesis in human SARS-CoV-2 infections ${ }^{17-19}$. Research on SARS-CoV and MERS-CoV has revealed that the interferon-mediated antiviral response is a double-edged sword which can induce both protective and pathogenic effects in humans. How Ca-DelMut infection induces the host immune response is of interest for understanding the molecular basis of its attenuation. To test if infection with Ca-DelMut may induce a different immune response from that elicited by wild type SARS-CoV-2, we examined interferon and cytokine expression in the lung tissues of virus infected hamsters. In contrast to infection with wild type HK-13 strain, we found that the Ca-DelMut variant does not provoke elevated levels of cytokines in infected hamsters (Figure 3 and S3). Aberrant activation of IL6 has been recognized as an important biomarker of disease severity in SARS-CoV-2 infected patients ${ }^{19-21}$. Remarkably, activation of IL-6 was only observed in HK-13 strain infected hamsters but not in those infected with Ca-DelMut variant. We then analyzed the adaptive immune response in hamsters previously infected with Ca-DelMut or wild type virus. Levels of receptor binding domain (RBD) specific antibodies in sera collected three weeks after infection were determined. Sera from both hamsters previously infected with Ca-DelMut or that had recovered from wild type virus (HK-13) ${ }^{22}$ infection showed robust induction of RBD specific antibodies (Figure 4A and S4A). Cell based neutralization assays also demonstrated strong neutralizing activity against the wild type virus strain HK-13 in sera collected from both Ca-DelMut variant and wild type SARS-CoV-2 virus (HK-13) infected hamsters (Figure 4B and S4B). These results indicate that infection with Ca-DelMut leads to an altered immune response which does not 
induce the elevated levels of proinflammatory cytokines seen in wild type SARS-CoV-2 virus infection. Nonetheless Ca-DelMut elicits a strong adaptive immune response, as demonstrated by robust neutralizing antibody production.

\section{Infection with Ca-DelMut confers full protection against SARS-CoV-2 infection with sterilizing immunity}

Because infection with Ca-DelMut causes no apparent disease in hamsters while inducing a strong neutralizing antibody response, we examined the potential of Ca-DelMut as a live attenuated virus vaccine to prevent SARS-CoV-2 virus infection and disease. Four weeks after infection with Ca-DelMut live attenuated virus, hamsters were re-challenged with wild type SARS-CoV-2 viruses. Two strains of SARS-CoV-2, HK-13 and HK-95, were used in the challenge experiment. HK-95 contains a D614G substitution in the spike protein, which has been suggested to bestow SARS-CoV-2 with higher infectivity in humans ${ }^{23}$. Negligible body weight loss was observed in Ca-DelMut vaccinated hamsters infected with either strain of wild type virus, whereas control hamsters not immunized with Ca-DelMut lost about 12-15 percent of body weight by day 5 post-infection (Figure 5A and S5). Analyses of virus replication in the lung and nasal turbinate tissues of re-challenged hamsters showed that Ca-DelMut infection provides sterilizing immunity against subsequent challenge with either HK-13 or HK-95 SARS-CoV-2, with the quantity of virus being either very low or below the level of detection in both lung and nasal tissues on days 2 and 5 post-infection (Figure 5B and S5). Histopathological analysis showed that mock-vaccinated hamster lungs collected at day 5 postinfection with either wild type virus strain showed extensive alveolar exudation and infiltration; bronchiolar epithelial cell death with luminal exudation and cell debris were also observed (Figure 6). In contrast, Ca-DelMut inoculated hamsters challenged with either strain of wild type SARS-CoV-2 virus only experienced mild regional alveolar septal infiltration and blood vessel congestion at days 2 and 5 post-infection. No other pulmonary histopathological changes 
160 were observed. Our experiment also showed a lower inoculum of Ca-DelMut $\left(1 \times 10^{3} \mathrm{pfu}\right)$ is 161 sufficient to provide full protection to the wild type virus challenge (Figure S6). These data 162 indicate that prior infection with live attenuated Ca-DelMut variant virus provides complete 163 protection against infection with wild type SARS-CoV-2 viruses in hamsters. 


\section{Discussion}

Coronaviruses are zoonotic pathogens with distinct cross species transmissibility ${ }^{24}$. Besides 229E, OC43, HKU-1 and NL63, which have long been circulating in humans, SARS$\mathrm{CoV}$, MERS-CoV and SARS-CoV-2 have jumped the species barrier to infect humans in recent years ${ }^{7}$. SARS-CoV disappeared in 2004, while MERS-CoV is restricted to a few countries in the Middle East with only sporadic human transmissions since $2012{ }^{25}$. SARS-CoV-2 virus utilizes the same cellular receptor as SARS-CoV, ACE2, for mediating human infection but has exhibited a distinctive infectivity and transmissibility profile since it was first recognized in humans in Wuhan, China, in December $2019^{6}$. There is strong interest in understanding how SARS-CoV-2 has acquired the unique ability to infect and transmit efficiently in humans. SARS-CoV-2 contains a PRRA polybasic motif not seen in the most closely related bat and pangolin coronaviruses currently known ${ }^{3,5,8}$. The presence of a polybasic cleavage site at the $\mathrm{S} 1 / \mathrm{S} 2$ junction of the spike protein of SARS-CoV-2 virus is considered a critical property for enhanced coronavirus infectivity in humans and zoonotic potential ${ }^{14,26}$. A peptide assay has shown that the polybasic cleavage site harbored in SARS-CoV-2 is more accessible to proteases which activate the coronavirus spike protein ${ }^{10}$. If the polybasic cleavage site is one of the essential elements providing SARS-CoV-2 with increased infectivity and pathogenicity in humans, removal of this determinant could logically attenuate SARS-CoV-2 into a mild respiratory virus similar to the less pathogenic common coronaviruses currently circulating. Our previous report on a panel of attenuated variants with deletions at the S1/S2 junction supports this contention ${ }^{15}$. This study has further characterized one such mutant, Ca-DelMut, showing that it has low pathogenicity and does not provoke an inflammatory response in hamsters and that it induces adaptative immunity protective against subsequent infection with more pathogenic SARS-CoV-2 strains. Ca-DelMut attenuated virus could also be a useful tool for studying SARS-CoV-2 replication, host tissue tropism and transmissibility. 

distinctive infectivity and pathogenicity displayed by SARS-CoV-2 is likely to be associated with the acquisition of a polybasic furin cleavage site in the protein which, together with enhanced binding affinity of the SARS-CoV-2 RBD for ACE2, would significantly broaden the tissue tropism of this virus ${ }^{14,27}$. Deregulated innate immunity during the early stage of infection in the upper respiratory tract may determine the subsequent outcome of dissemination to the lower respiratory tract and disease severity ${ }^{17,28}$. We found that Ca-DelMut replicates to comparable levels to wild type virus in the nasal turbinates but less effectively than wild type virus in the lungs (Figure 2C). Importantly, while replication of Ca-DelMut variant was observed in lung tissues, the elevated expression of proinflammatory cytokines elicited in wild 
216 immunity to prevent infection and alleviate disease severity and spread. Anti-SARS-CoV-2

217 antibodies are reported to decline rapidly in naturally infected individuals ${ }^{36,37}$. This study

218 showed that Ca-DelMut induces a different innate immune response to that observed in wild

219 type virus infections in an animal model and evokes sterilizing protective adaptative immunity

220 against challenge with wild type virus (Figure 4). Because Ca-DelMut is attenuated it does not

221 provoke proinflammatory cytokines which could interfere with the induction of adaptive

222 immunity. It is possible that the Ca-DelMut variant may be able to stimulate more balanced

223 and long-lasting immunity; further study is required to comprehensively compare the immune

224 profiles induced by Ca-DelMut and wild type SARS-CoV-2 viruses. The potential applications

225 of this attenuated SARS-CoV-2 virus should be explored and evaluated. However, given the

226 high replication efficiency and low pathogenicity of Ca-DelMut, it may be an ideal strain for

227 production of an inactivated vaccine, in addition to holding promise as a live attenuated vaccine. 


\section{Generation of cell adapted Del-mut (Ca-DelMut) virus}

231 Del-mut viruses were prepared as previously described ${ }^{15}$. To generate Ca-DelMut virus, the

232 Del-Mut-1 virus was serially passaged 10 times in Vero E6 cells at $33^{\circ} \mathrm{C}$ and then passaged at $23330^{\circ} \mathrm{C} 8$ times. For each passage, incubation time was 2-3 days, depending on the occurrence of 234 cytopathic effects. After the $18^{\text {th }}$ passage, the virus was amplified in a T75 Flask, then titered 235 by plaque assay and sequenced using the Sanger method.

\section{Growth kinetics}

237 Confluent Vero E6 or Calu-3 cells were infected at 0.01 moi with the indicated viruses and 238 incubated for 3 days. Virus supernatant was collected at the indicated time points. Virus titers 239 were determined by plaque assay using Vero E6 cells.

\section{$240 \quad$ Plaque assay}

241 Confluent Vero E6 cells in 6-well format were incubated with 10-fold serially diluted virus for $2421 \mathrm{~h}$. After adsorption, virus was discarded. Cells were washed and overlaid with $1 \%$ agarose in 243 DMEM and incubated for 3 days at $37^{\circ} \mathrm{C}$. Cells were fixed with $10 \%$ formaldehyde for 1 day. 244 Agarose gels were then removed and plaques stained with 1\% crystal violet and counted.

\section{Immunization and challenge of hamsters}

$246 \quad 7-8$ week old golden Syrian hamsters were anesthetized intraperitoneally with ketamine and 247 xylazine and then immunized intranasally with $1.25 \times 10^{5}$ pfu of Ca-DelMut or SZ-002 virus or mock immunized with PBS. Body weight and disease symptoms were monitored daily. At day 21, sera were collected from hamsters for anti-spike RBD IgG and neutralizing antibody determination. At day 28, Ca-DelMut- and mock-immunized hamsters were challenged with WT virus (HK-13 or HK-95) at a dose of $1 \times 10^{3}$ pfu. Body weight and disease symptoms were monitored daily and at days 2 and 5, lung and nasal turbinate tissues were collected for histopathology and virus titration by plaque assay. For low dose immunization, hamsters were 
immunized with $10^{3}$ pfu of either Ca-DelMut or WT HK-13 virus, or mock immunized with

PBS. All experiments involving SARS-CoV-2 were conducted in a biosafety level 3 laboratory.

256 All animal studies were approved by the Committee on the Use of Live Animals in Teaching and Research, The University of Hong Kong.

258

259

\section{Pathogenicity of Ca-DelMut and wild type SARS-CoV-2 virus in hamsters}

Hamsters were challenged intranasally with $1 \times 10^{3}$ or $1.25 \times 10^{5}$ pfu of Ca-DelMut or $1 \times 10^{3} \mathrm{pfu}$ of WT virus. Body weight and disease symptoms were monitored daily. At days 2 and 4, lung and nasal turbinate tissues were collected for histopathological study, determination of proinflammatory cytokine expression and virus titration.

\section{Neutralization assay}

Heat inactivated sera from Ca-DelMut challenged hamsters were 2-fold serially diluted in DMEM medium and incubated with $100 \mathrm{pfu}$ of the indicated virus at $37^{\circ} \mathrm{C}$ for $1 \mathrm{~h}$. The mix was added to confluent Vero E6 cells and incubated for 4 days at $37^{\circ} \mathrm{C}$. Naïve and WT challenged sera were used as controls. After 4 days, cytopathic effect (CPE) was detected by microscopy, with the neutralization endpoint being the highest serum dilution causing $50 \%$ inhibition of CPE.

\section{ELISA}

A hamster anti-spike RBD IgG detection kit (Wantai-Bio) was used to detect RBD specific antibodies. Procedures were conducted in accordance with the manual. Briefly, heat inactivated sera from Ca-DelMut challenged hamsters were 10-fold serially diluted and added to the plate and incubated at $37^{\circ} \mathrm{C}$ for 30 mins. Sera from mock- and WT-challenged hamsters were included as controls. The plate was washed 5 times and then incubated with secondary antibody reagent at $37^{\circ} \mathrm{C}$ for 30 mins. After washing, color development solution was added and the plate incubated at $37^{\circ} \mathrm{C}$ for 15 mins. Stop solution was added and absorbance at $450 \mathrm{~nm}$ measured. 
279

\section{Quantification of expression of proinflammatory cytokines and chemokines}

Expression of proinflammatory cytokines was quantified using a qRT-PCR technique similar to that described in a previous study ${ }^{38}$. Briefly, total RNA was extracted from hamster lungs using RNAzol RT reagent (MRC) according to the manual. cDNA was synthesized using a High Capacity cDNA Reverse Transcription Kit (Invitrogen) and oligo dT primers following the protocol provided. qPCR was performed using SYBR Premix Ex Taq (Takara) reagent and gene specific primers in an LC480 PCR machine (Roche). PCR conditions were as follows: initial denaturation: $95^{\circ} \mathrm{C}$ for $5 \mathrm{~min}, 45$ cycles of amplification: $95^{\circ} \mathrm{C}$ for $10 \mathrm{~s}, 60^{\circ} \mathrm{C}$ for $10 \mathrm{~s}$, $72^{\circ} \mathrm{C}$ for $10 \mathrm{~s}$, and melting curve analysis: $65^{\circ} \mathrm{C}$ to $97^{\circ} \mathrm{C}$ at $0.1^{\circ} \mathrm{C} / \mathrm{s}$. Expression of target genes was normalized to the internal reference gene (hamster $\gamma$-actin) and the comparative $\mathrm{Ct}$ (2$\Delta \Delta \mathrm{Ct}$ ) method utilized to calculate the cytokine expression profile.

\section{Histopathology}

Organs were fixed in 10\% PBS buffered formalin and processed into paraffin-embedded blocks. Tissue sections were stained with haematoxylin and eosin (H\&E) and examined by light microscopy as in a previous study ${ }^{38}$.

\section{Acknowledgements}

The authors would like to thank Dr Jane Rayner for critical reading and editing of the manuscript. This study is partly supported by the Theme-Based Research Scheme (T11/707/15) and General Research Fund (17107019) of the Research Grants Council, Hong Kong Special Administrative Region and the Sanming Project of Medicine in Shenzhen, China (No. SZSM201911014).

\section{Author Contributions}


Y. and H.C. analyzed the data; P.W. and H.C. wrote the paper.

\section{Competing Interests statement}

The authors declare no conflict of interests.

\section{References}

1. Wu, F., et al. A new coronavirus associated with human respiratory disease in China. Nature 579, 265-269 (2020).

2. Lu, R., et al. Genomic characterisation and epidemiology of 2019 novel coronavirus: implications for virus origins and receptor binding. Lancet 395, 565-574 (2020).

3. Lam, T.T., et al. Identifying SARS-CoV-2-related coronaviruses in Malayan pangolins. Nature 583, 282-285 (2020).

4. Zhang, T., Wu, Q. \& Zhang, Z. Probable Pangolin Origin of SARS-CoV-2 Associated with the COVID-19 Outbreak. Curr Biol 30, 1578 (2020).

5. Zhou, H., et al. A Novel Bat Coronavirus Closely Related to SARS-CoV-2 Contains Natural Insertions at the S1/S2 Cleavage Site of the Spike Protein. Curr Biol 30, 2196-2203 e2193 (2020).

6. Zhou, P., et al. A pneumonia outbreak associated with a new coronavirus of probable bat origin. Nature 579, 270-273 (2020).

7. de Wit, E., van Doremalen, N., Falzarano, D. \& Munster, V.J. SARS and MERS: recent insights into emerging coronaviruses. Nat Rev Microbiol 14, 523-534 (2016).

8. Andersen, K.G., Rambaut, A., Lipkin, W.I., Holmes, E.C. \& Garry, R.F. The proximal origin of SARS-CoV-2. Nat Med 26, 450-452 (2020).

9. Matsuyama, S., Ujike, M., Morikawa, S., Tashiro, M. \& Taguchi, F. Protease-mediated enhancement of severe acute respiratory syndrome coronavirus infection. Proc Natl Acad Sci U S A 102, 12543-12547 (2005).

10. Jaimes, J.A., Millet, J.K. \& Whittaker, G.R. Proteolytic Cleavage of the SARS-CoV-2 Spike Protein and the Role of the Novel S1/S2 Site. iScience 23, 101212 (2020).

11. Kawaoka, Y. \& Webster, R.G. Sequence requirements for cleavage activation of influenza virus hemagglutinin expressed in mammalian cells. Proc Natl Acad Sci U S A 85, 324-328 (1988).

12. Ito, T., et al. Generation of a highly pathogenic avian influenza A virus from an avirulent field isolate by passaging in chickens. J Virol 75, 4439-4443 (2001).

13. Alexander, D.J. \& Brown, I.H. History of highly pathogenic avian influenza. Rev Sci Tech 28, 19-38 (2009).

14. Hoffmann, M., Kleine-Weber, H. \& Pohlmann, S. A Multibasic Cleavage Site in the Spike Protein of SARS-CoV-2 Is Essential for Infection of Human Lung Cells. Mol Cell 78, 779-784 e775 (2020).

15. Lau, S.Y., et al. Attenuated SARS-CoV-2 variants with deletions at the S1/S2 junction. Emerg Microbes Infect 9, 837-842 (2020).

16. Wong, Y.C., et al. Natural transmission of bat-like SARS-CoV-2PRRA variants in COVID-19 patients. Clin Infect Dis (2020).

17. Zhou, Z., et al. Heightened Innate Immune Responses in the Respiratory Tract of COVID-19 Patients. Cell Host Microbe 27, 883-890 e882 (2020). 
18. Giamarellos-Bourboulis, E.J., et al. Complex Immune Dysregulation in COVID-19 Patients with Severe Respiratory Failure. Cell Host Microbe 27, 992-1000 e1003 (2020).

19. Mazzoni, A., et al. Impaired immune cell cytotoxicity in severe COVID-19 is IL-6 dependent. J Clin Invest (2020).

20. Chen, X., et al. Detectable serum SARS-CoV-2 viral load (RNAaemia) is closely correlated with drastically elevated interleukin 6 (IL-6) level in critically ill COVID-19 patients. Clin Infect Dis (2020).

21. Luo, M., et al. IL-6 and CD8+ T cell counts combined are an early predictor of in-hospital mortality of patients with COVID-19. JCI Insight 5(2020).

22. Chan, J.F., et al. A familial cluster of pneumonia associated with the 2019 novel coronavirus indicating person-to-person transmission: a study of a family cluster. Lancet 395, 514-523 (2020).

23. Korber, B., et al. Tracking Changes in SARS-CoV-2 Spike: Evidence that D614G Increases Infectivity of the COVID-19 Virus. Cell (2020).

24. Menachery, V.D., Graham, R.L. \& Baric, R.S. Jumping species-a mechanism for coronavirus persistence and survival. Curr Opin Virol 23, 1-7 (2017).

25. Chan, J.F., et al. Middle East respiratory syndrome coronavirus: another zoonotic betacoronavirus causing SARS-like disease. Clin Microbiol Rev 28, 465-522 (2015).

26. Menachery, V.D., et al. Trypsin Treatment Unlocks Barrier for Zoonotic Bat Coronavirus Infection. J Virol 94(2020).

27. Wrobel, A.G., et al. SARS-CoV-2 and bat RaTG13 spike glycoprotein structures inform on virus evolution and furin-cleavage effects. Nat Struct Mol Biol 27, 763-767 (2020).

28. Hou, Y.J., et al. SARS-CoV-2 Reverse Genetics Reveals a Variable Infection Gradient in the Respiratory Tract. Cell 182, 429-446 e414 (2020).

29. Guan, Y., et al. Isolation and characterization of viruses related to the SARS coronavirus from animals in southern China. Science 302, 276-278 (2003).

30. Su, Y.C.F., et al. Discovery and Genomic Characterization of a 382-Nucleotide Deletion in ORF7b and ORF8 during the Early Evolution of SARS-CoV-2. mBio 11(2020).

31. Gong, Y.N., et al. SARS-CoV-2 genomic surveillance in Taiwan revealed novel ORF8-deletion mutant and clade possibly associated with infections in Middle East. Emerg Microbes Infect 9, 1457-1466 (2020).

32. Gao, Q., et al. Development of an inactivated vaccine candidate for SARS-CoV-2. Science 369, 77-81 (2020).

33. Corbett, K.S., et al. Evaluation of the mRNA-1273 Vaccine against SARS-CoV-2 in Nonhuman Primates. N Engl J Med (2020).

34. Mercado, N.B., et al. Single-shot Ad26 vaccine protects against SARS-CoV-2 in rhesus macaques. Nature (2020).

35. Folegatti, P.M., et al. Safety and immunogenicity of the ChAdOx1 nCoV-19 vaccine against SARS-CoV-2: a preliminary report of a phase $1 / 2$, single-blind, randomised controlled trial. Lancet (2020).

36. Long, Q.X., et al. Clinical and immunological assessment of asymptomatic SARS-CoV-2 infections. Nat Med 26, 1200-1204 (2020).

37. Yin, S., et al. Longitudinal anti-SARS-CoV-2 antibody profile and neutralization activity of a COVID-19 patient. J Infect 81, e31-e32 (2020).

38. Chan, J.F., et al. Simulation of the clinical and pathological manifestations of Coronavirus Disease 2019 (COVID-19) in golden Syrian hamster model: implications for disease pathogenesis and transmissibility. Clin Infect Dis (2020). 
Figure 1. Schematic diagram of the SARS-CoV-2 genome showing the deletion and mutations of Ca-DelMut. Del-Mut-1 virus ${ }^{15}$ was serially passaged in Vero E6 cells at $33^{\circ} \mathrm{C}$ (10 passages) and $30^{\circ} \mathrm{C}\left(8\right.$ passages). Virus from the $18^{\text {th }}$ passage was designated as Ca-DelMut live attenuated SARS-CoV-2 virus and amplified to prepare a virus stock. Ca-DelMut was sequenced by the Sanger method; mutations are shown in the diagram and in Supplementary Table 1.

Figure 2. Replication efficiency of Ca-DelMut in vitro and in vivo. (A) Vero E6 or Calu-3 cells were infected with Ca-DelMut and other viruses at 0.01 moi and cultured at $37^{\circ} \mathrm{C}$. At the indicated time points, supernatants were collected, and virus titer determined by plaque assay in Vero E6 cells. (B) Ca-DelMut infection in hamsters. Hamsters were infected intranasally with either Ca-DelMut or wild type (WT) viruses at different doses, as indicated. Body weight was monitored for 5 days. (C) Replication of Ca-DelMut in lung and nasal turbinate tissues. After virus challenge $\left(1 \times 10^{3} \mathrm{pfu}\right)$, lung and nasal turbinate tissues were collected from hamsters at days 2 and 4, then homogenized and virus titer determined. Error bars represent mean \pm s.d. $(\mathrm{n}=3)$. Statistical comparisons between means were performed by Student's t-test: $* * * \mathrm{p}<0.001$, $* * \mathrm{p}<0.01, * \mathrm{p}<0.05, \mathrm{NS}$ : not significant.

Figure 3. Proinflammatory cytokine response profiles in Ca-DelMut and wild type virus infected hamsters. Hamsters were infected intranasally with $1 \times 10^{3}$ pfu of either Ca-DelMut or WT HK-13 virus. At days 2 and 4, RNA was extracted from lung tissues of infected hamsters and cDNA synthesized using oligo dT primers. Expression of different proinflammatory cytokines was examined by qPCR, normalized to an internal reference gene (hamster $\gamma$-actin), and the comparative $\mathrm{Ct}(2-\Delta \Delta \mathrm{Ct})$ method utilized to calculate the cytokine expression profile. Statistical comparisons between means were performed by Student's t-test: $* * * * p<0.0001$, $* * * \mathrm{p}<0.001, * * \mathrm{p}<0.01, * \mathrm{p}<0.05$, ns: not significant. 
Figure 4. Antibodies induced by Ca-DelMut immunization of hamsters. Hamsters were immunized intranasally with $1.25 \times 10^{5}$ pfu of either Ca-DelMut or wild type virus (HK-001a)

423 virus ${ }^{15,22}$, or mock immunized. At day 21, blood was collected from hamsters and tested for (A) anti-S1 RBD specific IgG titers and (B) neutralization activity against the HK-13 virus strain.

425 Error bars represent mean \pm s.d. $(n=3)$. LOD: level of detection. Statistical comparisons 426 between means were performed by Student's t-test: * $\mathrm{p}<0.05$, ns: not significant

427 Figure 5. Ca-DelMut immunization protection against WT virus challenge in hamsters.

428 Hamsters were inoculated with $1.25 \times 10^{5} \mathrm{pfu}$ Ca-DelMut or mock immunized. At day 28 after immunization, hamsters were challenged with $1 \times 10^{3}$ pfu of either HK-13 or HK-95 virus. (A) Body weight and disease symptoms were monitored for 5 days. (B) At days 2 and 5 postinfection, lungs and nasal turbinate tissues were collected for virus titration and histopathological study. Error bars represent mean \pm s.d. $(n=3)$.

Figure 6. Histopathological analysis of lung pathology in WT virus challenged Ca-

DelMut- and mock-immunized hamsters. At day 28 after immunization, hamsters were challenged with $1 \times 10^{3}$ pfu of either HK-13 or HK-95 virus. At days 2 and 5 post-infection, lungs were collected, fixed, processed into paraffin blocks and sections H\&E stained.

(a) Challenge with HK-13. Day 2: Mock-vaccinated hamster lungs showed bronchiolar epithelial cell death and the bronchiolar lumen filled with exudate and cell debris (arrow). Diffuse alveolar infiltration and focal hemorrhage were also seen (arrowheads). Ca-DelMutvaccinated hamster lungs showed regional alveolar septal infiltration and blood vessel congestion (arrowhead), but no obvious bronchiolar epithelial cell death (arrow). Day 5: Lungs of mock-vaccinated hamsters showed severe alveolar infiltration and exudation (arrowheads), as well as bronchiolar luminal exudation (arrow). Vaccinated hamster lungs showed focal 444 alveolar septal infiltration (arrowheads), while the bronchiolar epithelium appeared normal 445 with no luminal secretion or cell debris (arrows). 
446 (b) Challenge with HK-95. Day 2: Mock-vaccinated hamster lungs showed bronchiolar 447 epithelial cell death with luminal cell debris (arrow) and diffuse alveolar infiltration with focal 448 hemorrhage and exudation (arrowheads), with a medium sized blood vessel showing severe 449 endotheliitis (open arrow). Vaccinated hamster lungs showed regional alveolar septal 450 infiltration and blood vessel congestion (arrowhead); a blood vessel appeared to be normal 451 (open arrow), and no obvious bronchiolar epithelial cell death was observed (arrows). Day 5: 452 Lungs of mock-vaccination control hamsters showed severe alveolar infiltration and exudation 453 (arrowheads) and bronchiolar luminal cell debris (arrow). Vaccinated hamster lungs showed 454 focal alveolar septal infiltration (arrowheads), while the bronchiolar epithelium appeared 455 normal without luminal secretion (arrows). Scale bar: $100 \mu \mathrm{m}$. 


\section{Figure 1}

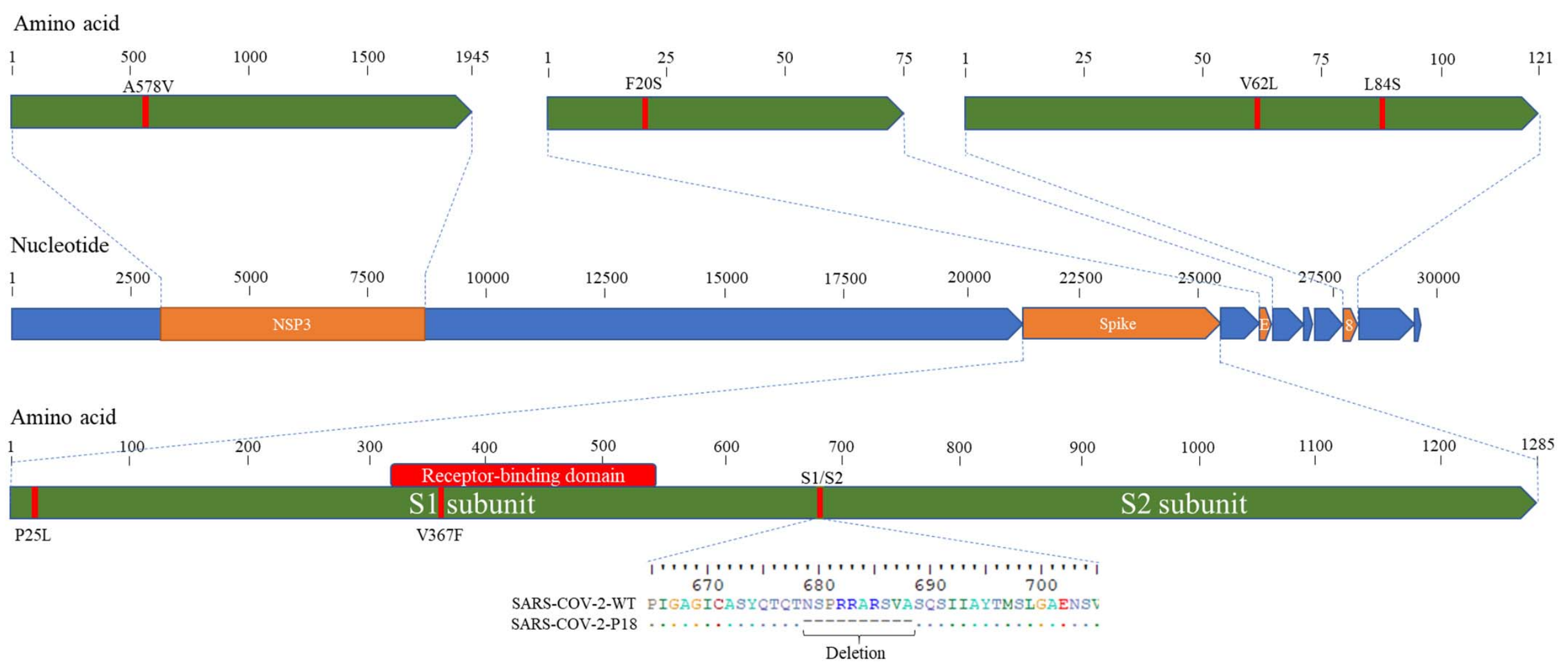


Figure 2

a

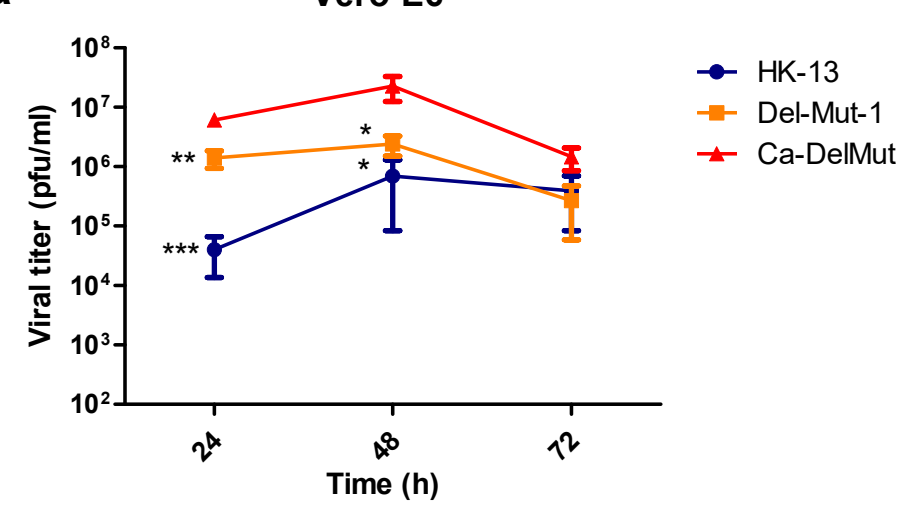

Calu-3

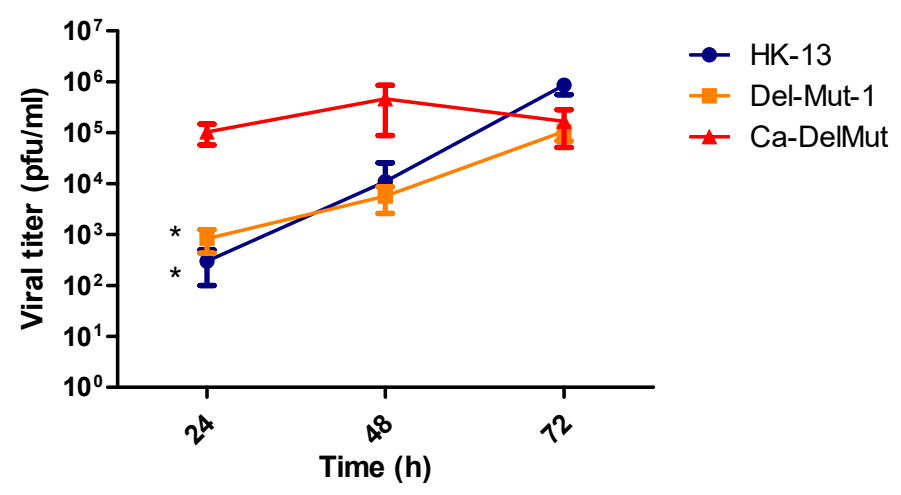

C

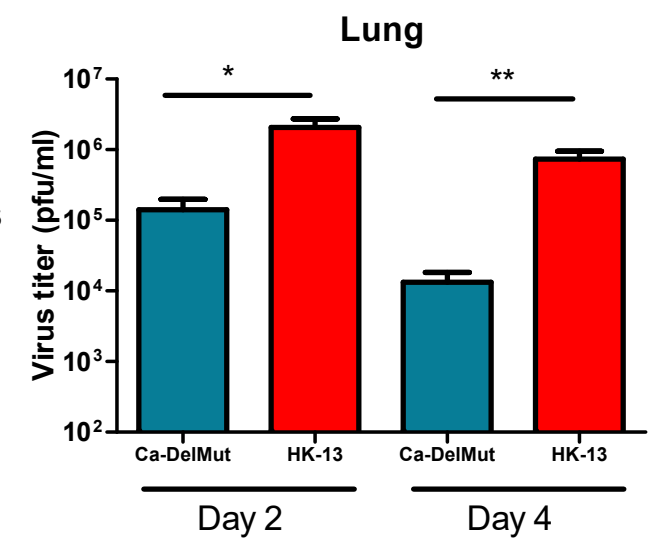

Nasal turbinates

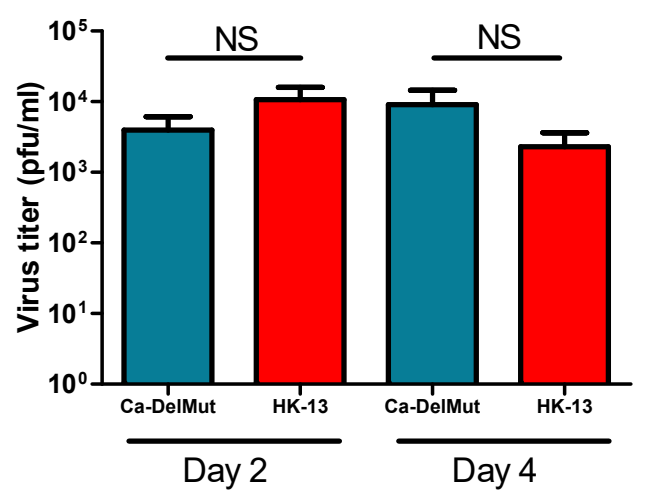


Figure 3

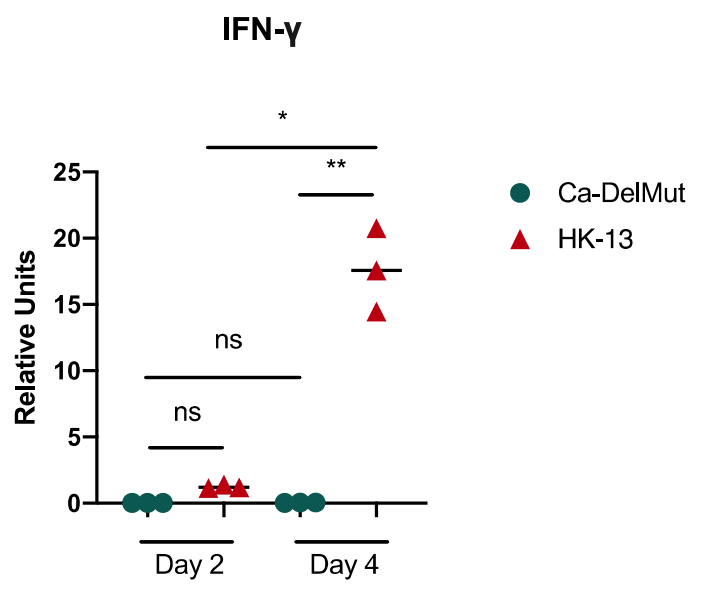

CCR4

IL-10

IL-21

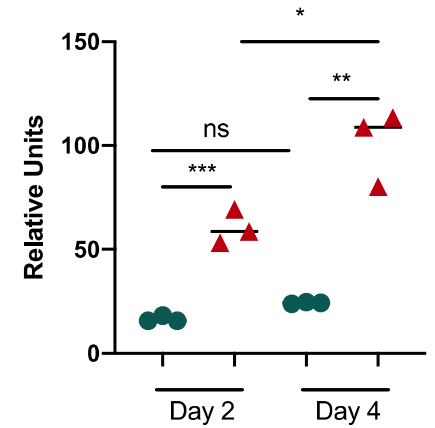

- Ca-DelMut

\ HK-13
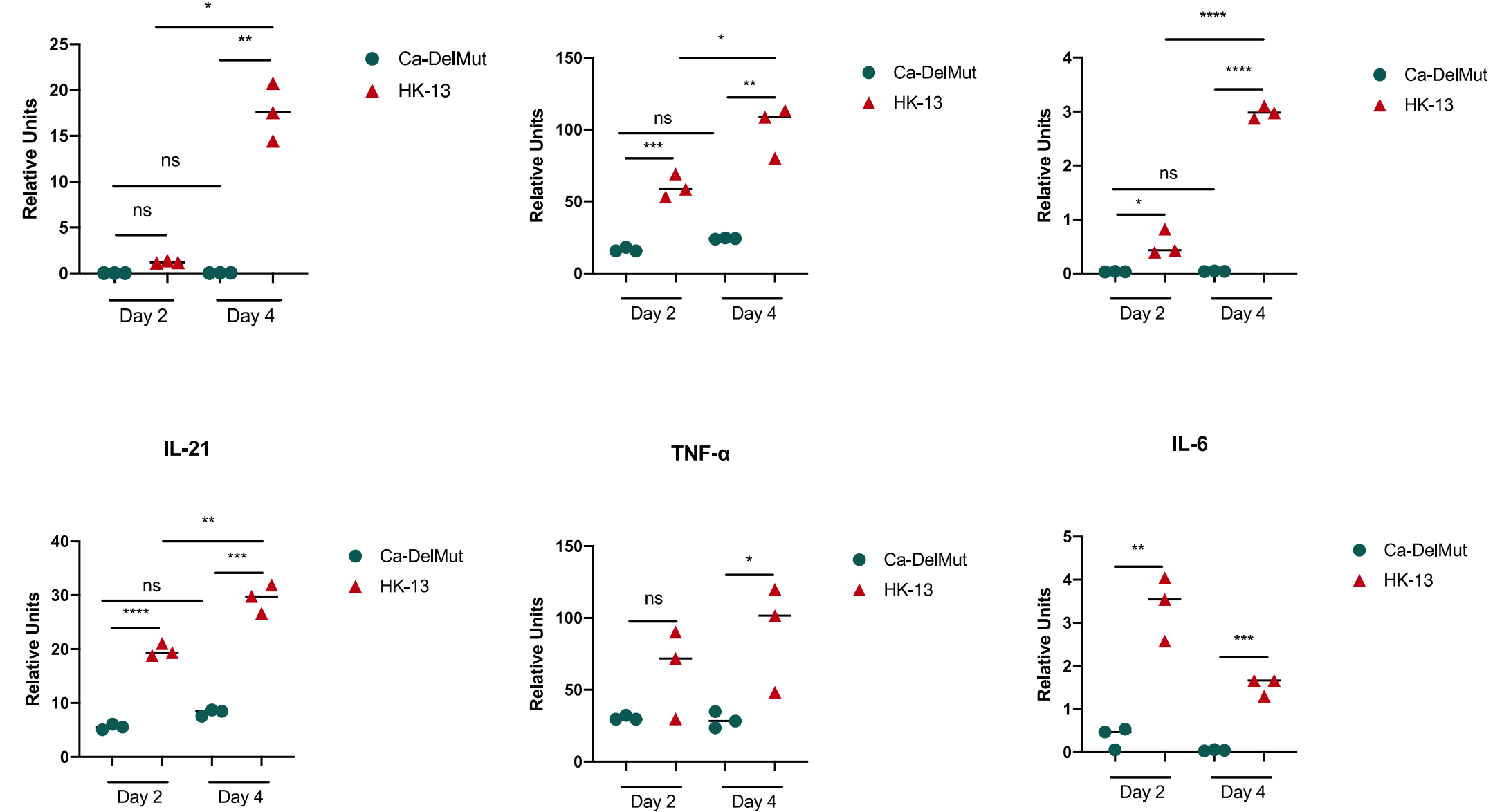

TNF- $\alpha$

IL-6
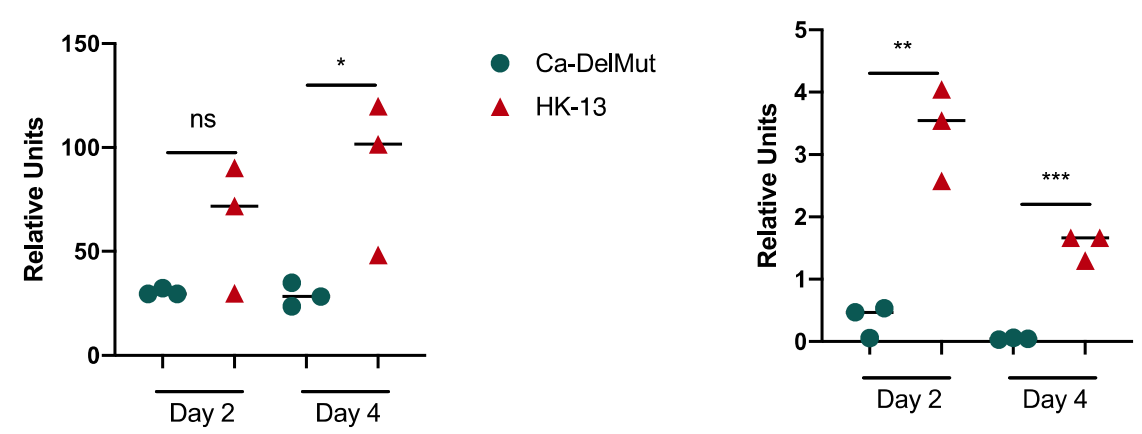

- Ca-DelMut

- Ca-DelMu

- HK-13

- Ca-DelMut

A HK-13

\ HK-13 
Figure 4

a

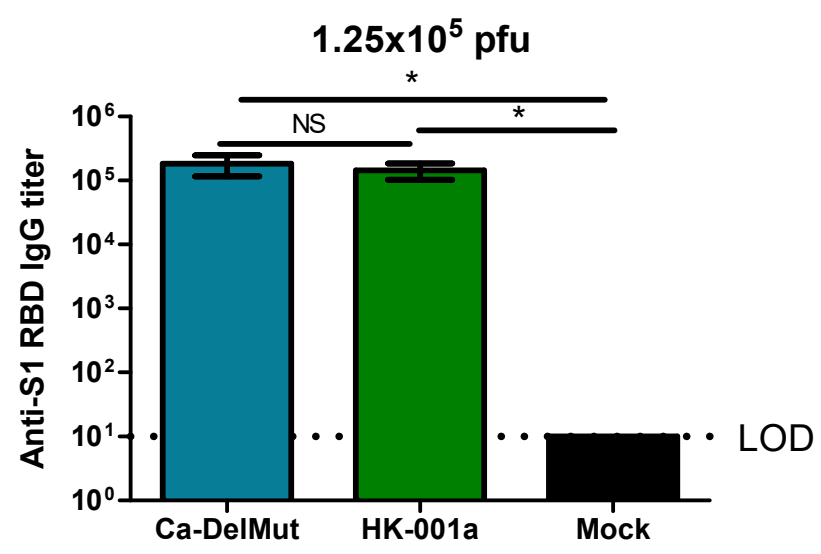

b

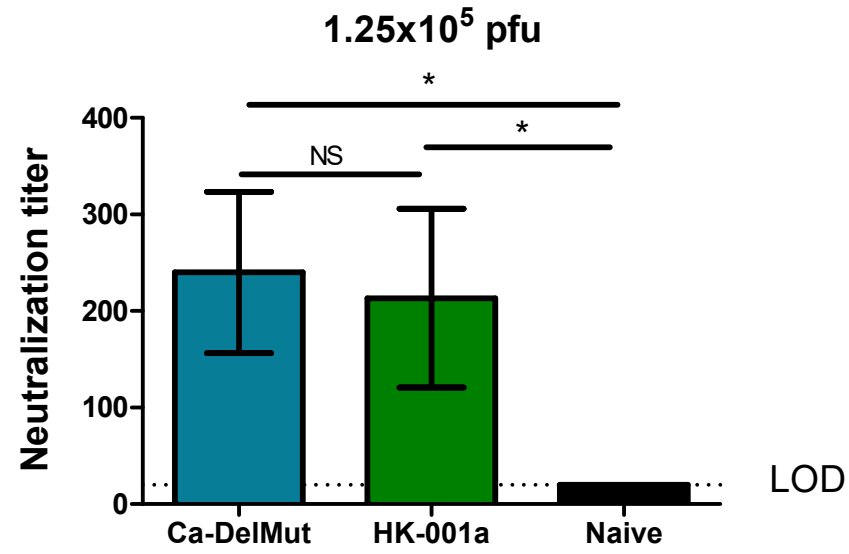


Figure 5

A

HK-13

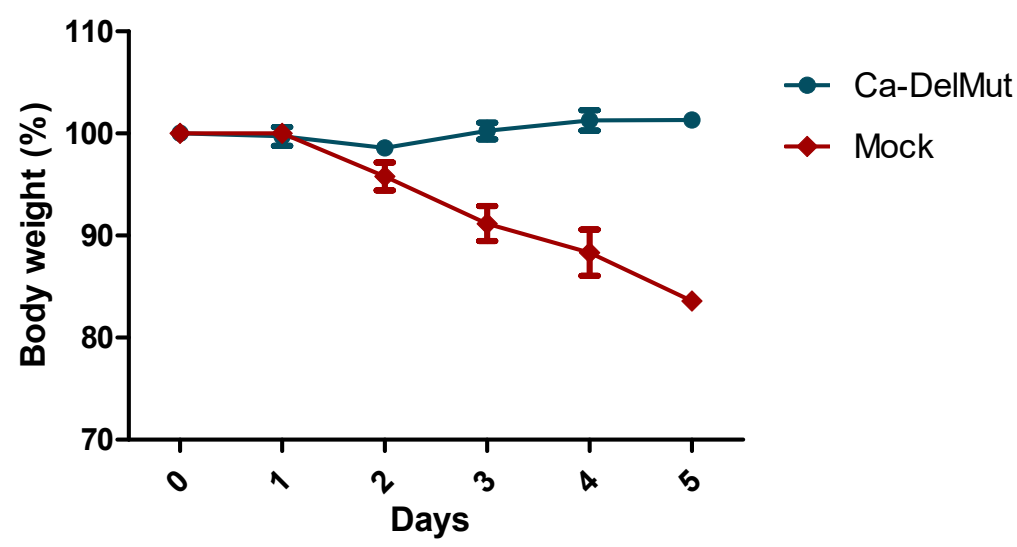

B

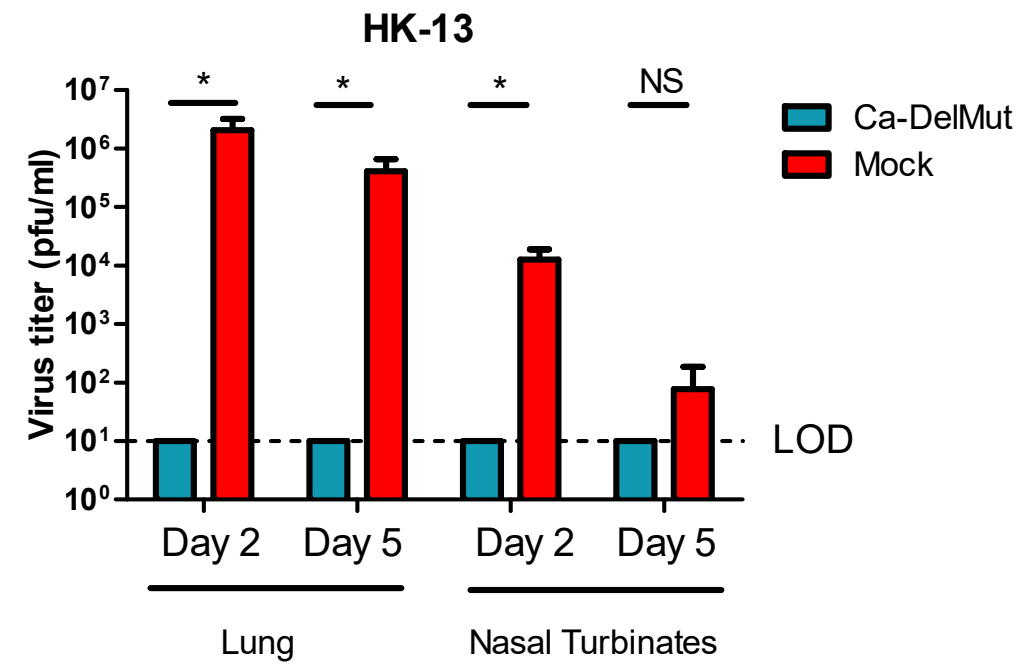

HK-95

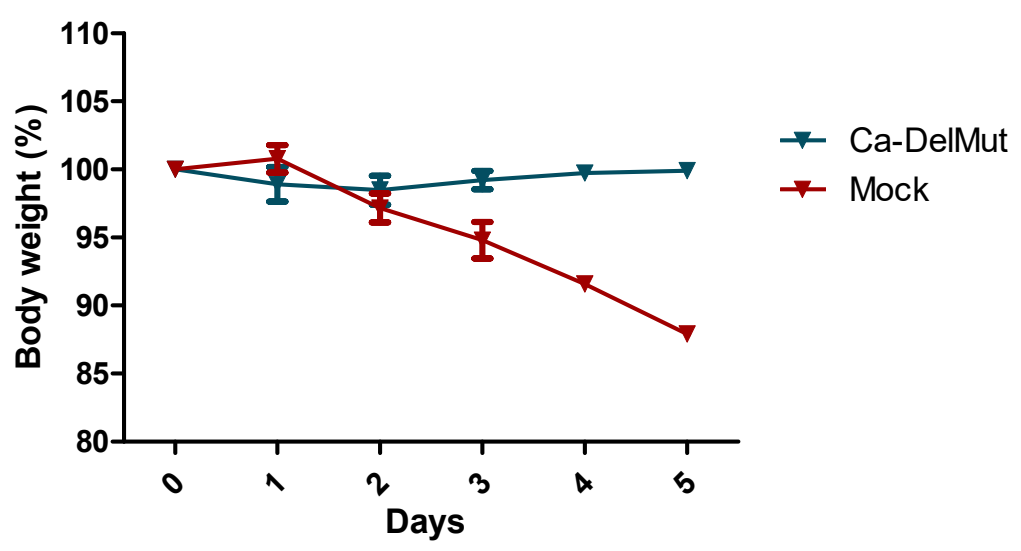

HK-95

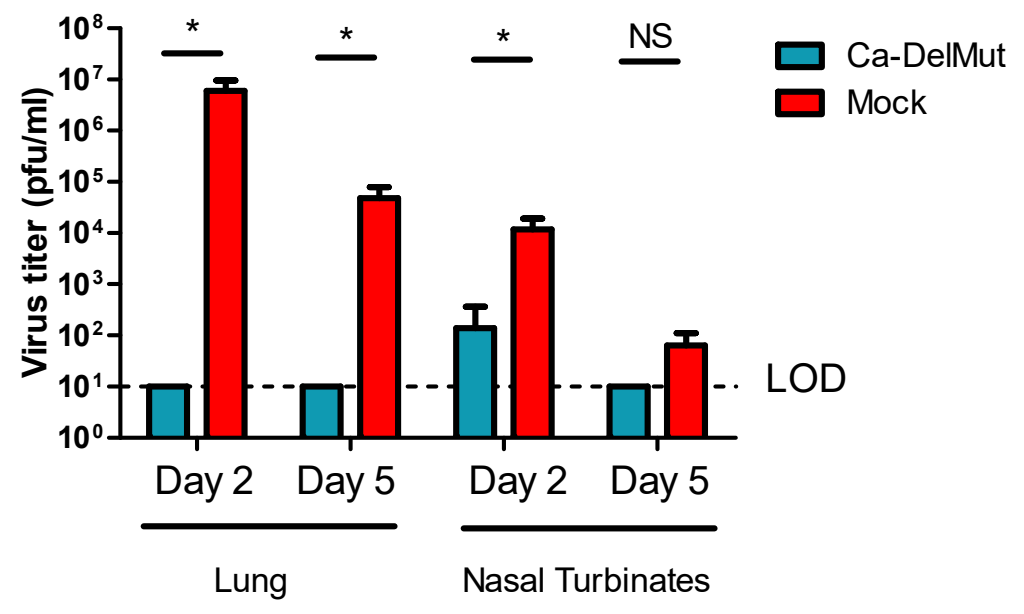




\section{Figure 6}

HK-13
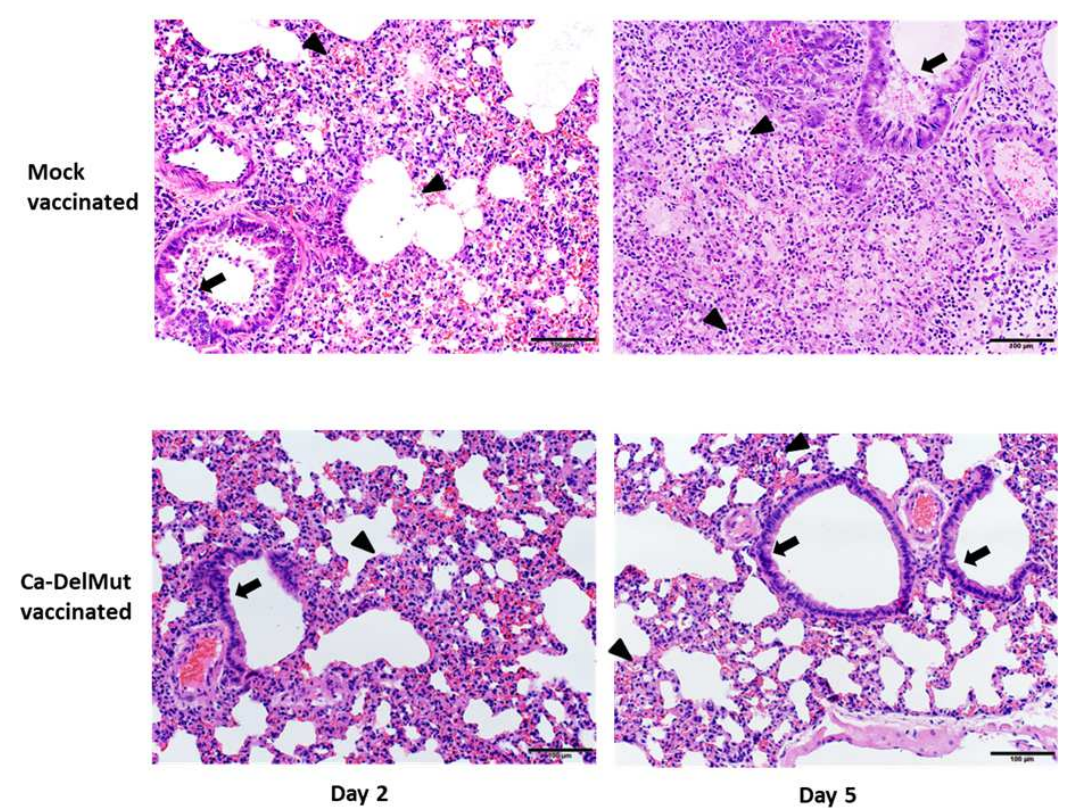

HK-95
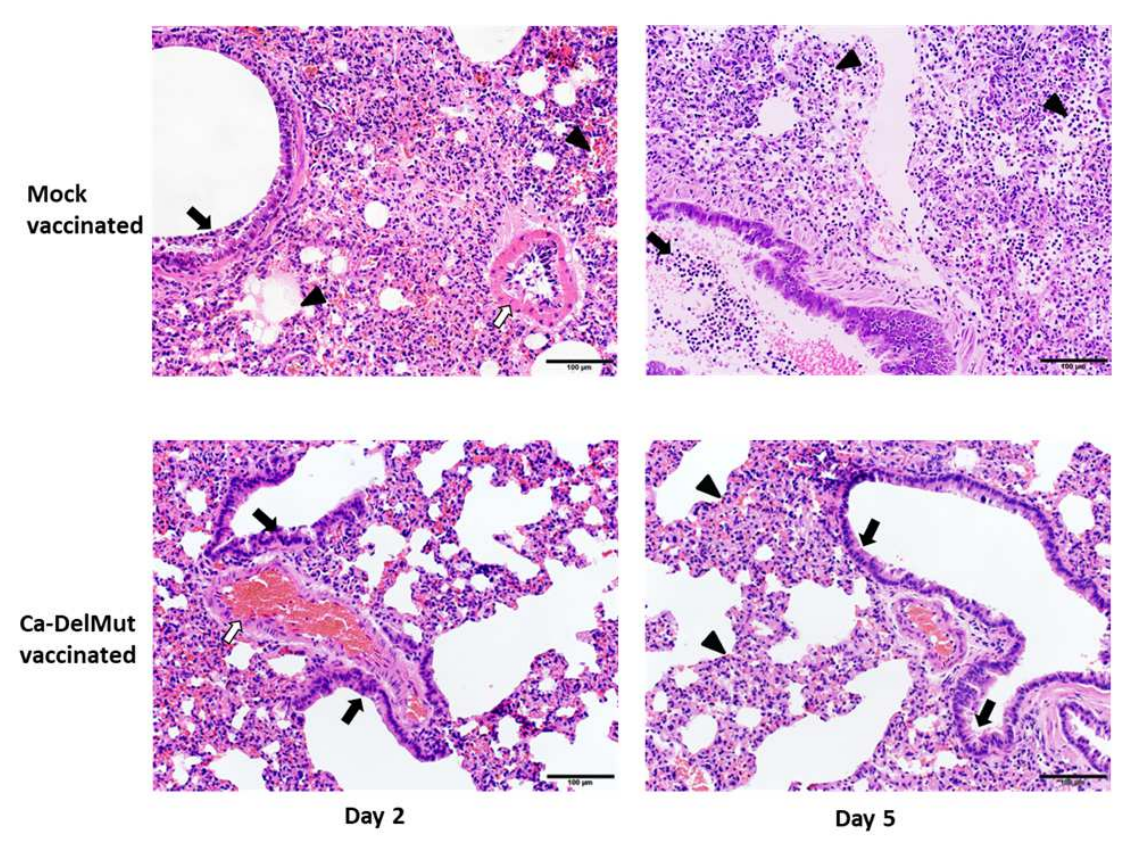
Pathogenicity, immunogenicity, and protective ability of an attenuated SARS-CoV-2 variant with a deletion at the $\mathrm{S1/S2}$ junction of the spike protein

Supplementary Table 1*

Deletion and mutations in Ca-DelMut virus genome compared to Wuhan-Hu-1

\begin{tabular}{|c|c|c|c|c|c|}
\hline \multirow{2}{*}{$\begin{array}{l}\text { Nucleotide } \\
\text { coordinate }\end{array}$} & \multirow{2}{*}{ Wuhan-Hu-1 } & \multicolumn{4}{|c|}{ Ca-DelMut } \\
\hline & & Nucleotide & Amino acid & Protein & ORF \\
\hline 1663 & $\mathrm{C}$ & $\mathbf{T}$ & No change & NSP2 & ORF1a \\
\hline 4455 & $\mathrm{C}$ & $\mathbf{T}$ & A578V & NSP3a & ORF1a \\
\hline 8782 & $\mathrm{C}$ & $\mathbf{T}$ & No change & NSP4 & ORF1a \\
\hline 21636 & $\mathrm{C}$ & $\mathbf{T}$ & P25L & $\mathrm{S}$ & $\mathrm{S}$ \\
\hline 22661 & $\mathrm{G}$ & $\mathbf{T}$ & V367F & $S$ & $\mathrm{~S}$ \\
\hline $23598-23627$ & No deletion & $\begin{array}{l}\text { 30-base-pair } \\
\text { deletion }\end{array}$ & 10 aa deletion & S & S \\
\hline 24034 & $\mathrm{C}$ & $\mathbf{T}$ & No change & $\mathrm{S}$ & $\mathrm{S}$ \\
\hline 26303 & $\mathrm{~T}$ & $\mathrm{C}$ & F20S & $\mathrm{E}$ & $\mathrm{E}$ \\
\hline 26729 & $\mathrm{~T}$ & $\mathrm{C}$ & No change & $M$ & M \\
\hline 28077 & G & $\mathrm{C}$ & V62L & ORF8 & ORF8 \\
\hline 28144 & $\mathrm{~T}$ & $\mathrm{C}$ & L84S & ORF8 & ORF8 \\
\hline
\end{tabular}

*Details of deletion and mutations in the Ca-DelMut variant with reference to the WuhanHu-1 SARS-CoV-2 strain (Wu et al., 2020)

Wu, F., Zhao, S., Yu, B., Chen, Y.M., Wang, W., Song, Z.G., Hu, Y., Tao, Z.W., Tian, J.H., Pei, Y.Y., et al. (2020). A new coronavirus associated with human respiratory disease in China. Nature 579, 265-269. 
Figure S1

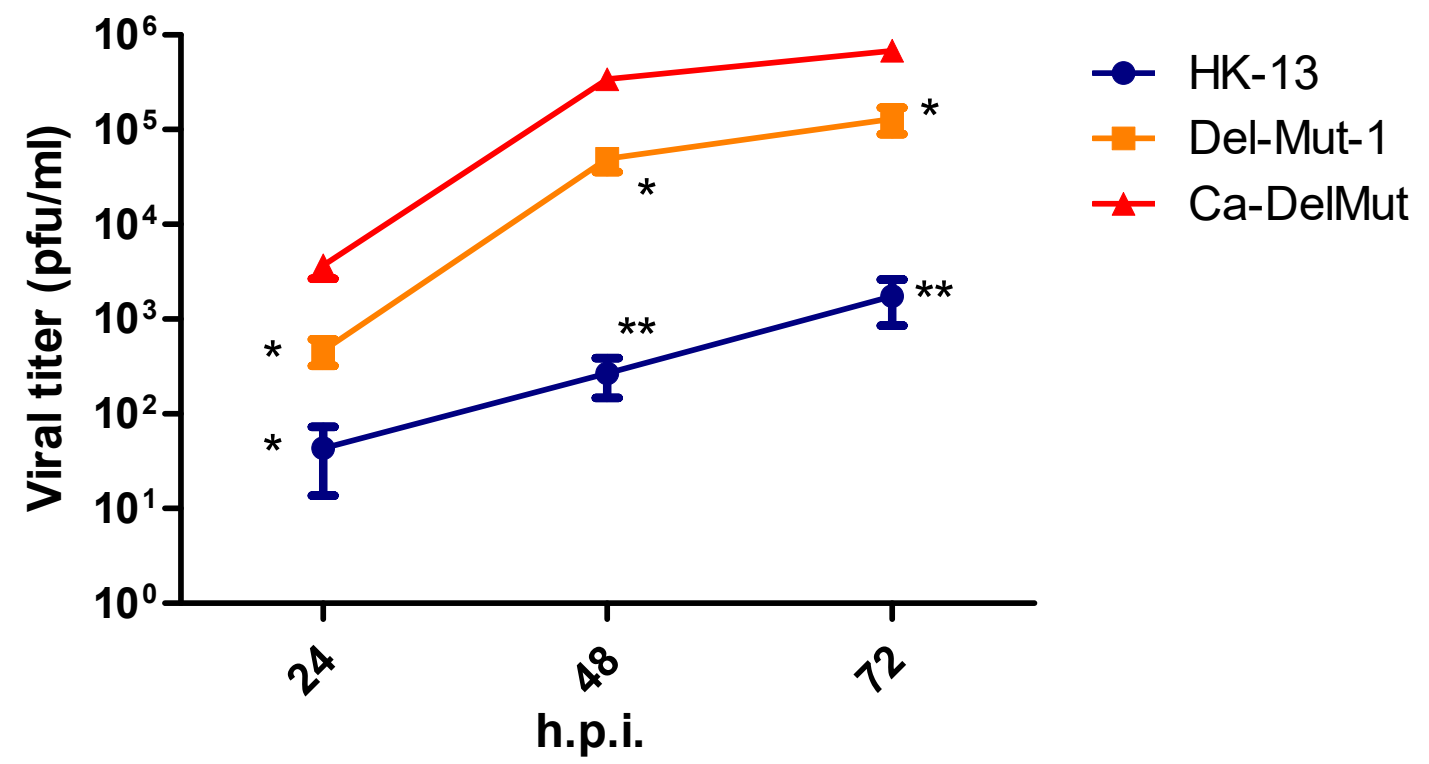

Figure S1. Ca-DelMut exhibits a cold-adapted phenotype in vitro. Vero E6 cells were infected with Ca-DelMut and other viruses at 0.01 moi and incubated at $30^{\circ} \mathrm{C}$. At the indicated time points, supernatants were collected and virus titer determined by plaque assay. Error bars represent mean \pm s.d. $(n=3)$. h.p.i.: hours post infection. 


\section{Figure S2}

$\mathbf{a}$

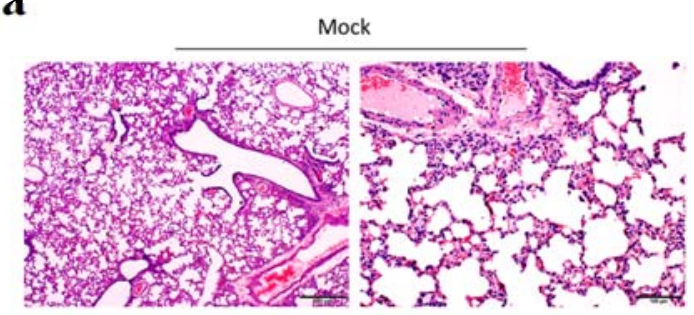

Day 2
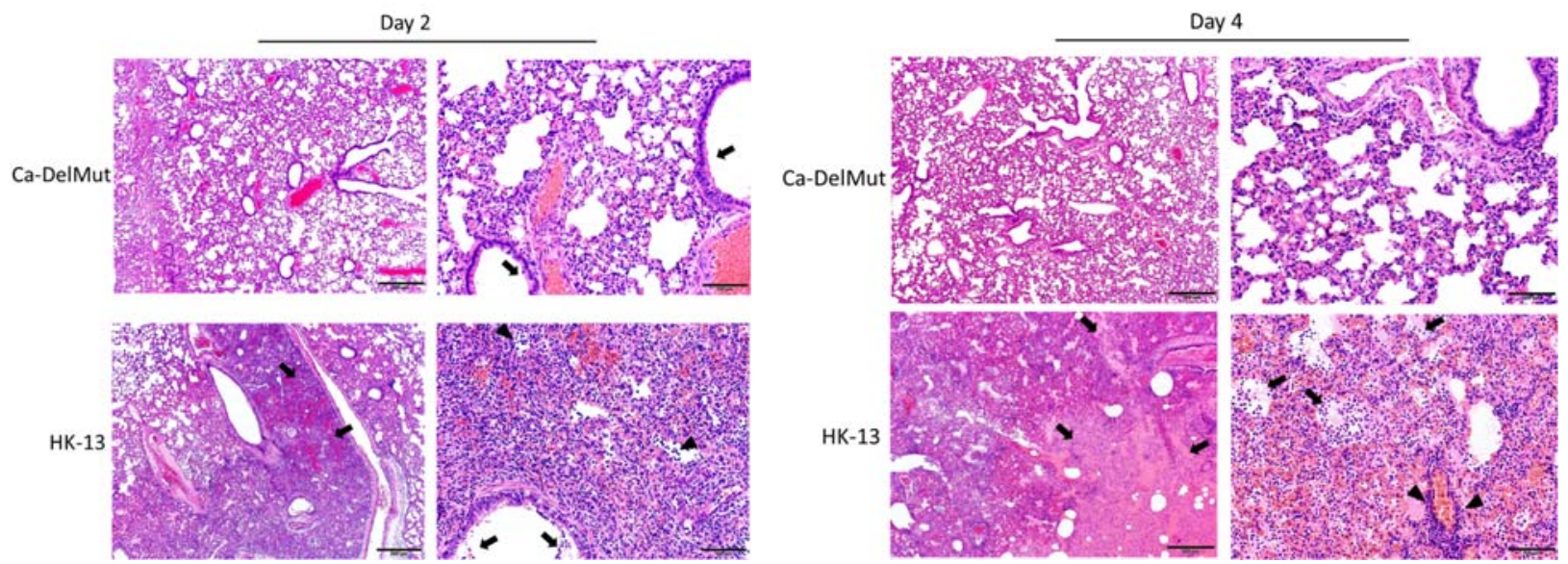

b
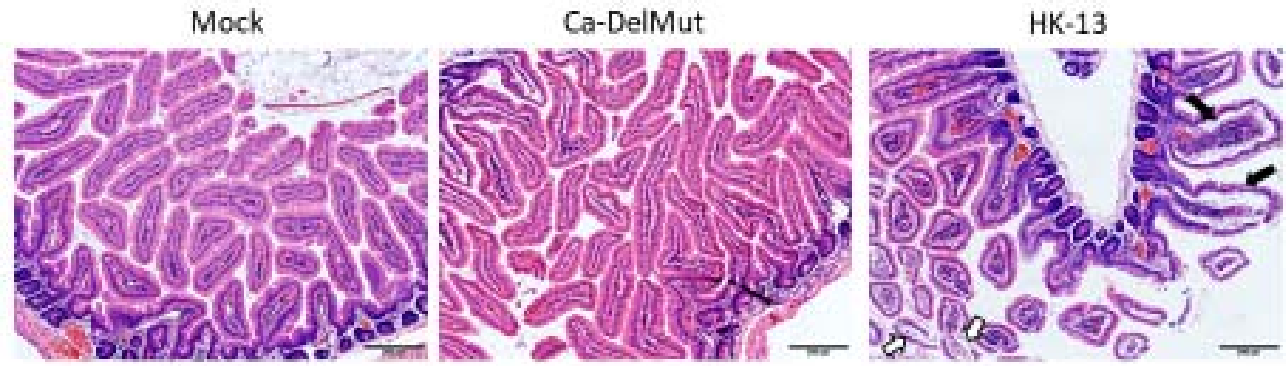

Figure S2. Infection with Ca-DelMut causes only mild pathological changes. Hamsters were

infected with $1 \times 10^{3}$ pfu of either Ca-DelMut or HK-13 (WT), or mock infected. At days 2 and 4 post-infection, lungs and small intestine were collected and fixed in $10 \%$ formalin, and then processed into paraffin blocks and sections H\&E stained.

(a) The top panel shows normal lung structures in the lungs of mock-infected control hamsters at 4x (left) and 20x (right) magnification. At day 2 after infection with Ca-DelMut, lung tissues showed only mild regional alveolar septal infiltration and blood vessel congestion. No obvious bronchiolar epithelium desquamation or luminal debris (arrows), and no alveolar space infiltration or exudation were observed. At day 4, no deleterious progression of histopathology was observed.

For WT virus at day 2 post-infection, the low magnification image (left) showed regional lung consolidation and focal pulmonary hemorrhage (arrows). The higher magnification image 
(right) showed massive alveolar space infiltration (arrowheads) and hemorrhage, with a little bronchiolar luminal cell debris (arrows) visible. At day 4 post WT-infection, the low magnification image (left) showed intensive alveolar exudation, infiltration and hemorrhage resulting in pulmonary consolidation (arrows), while the higher magnification image (right) showed intensive protein rich exudates filling the alveolar space (arrows), in addition to massive infiltration and alveolar hemorrhage; a blood vessel shows moderate infiltration (arrowheads).

(b) Representative histological images of hamster small intestines. The left-most image shows mock-infected control hamster small intestinal villi with normal structure. At day 4 post infection with Ca-DelMut, no apparent histopathological changes were detected in the small intestine (middle). For WT virus infection, small intestinal lamina propria blood vessel congestion, infiltration and edema resulting in swelling of the villi (solid arrows) and enterocyte desquamation (open arrows) were observed. 


\section{Figure S3}
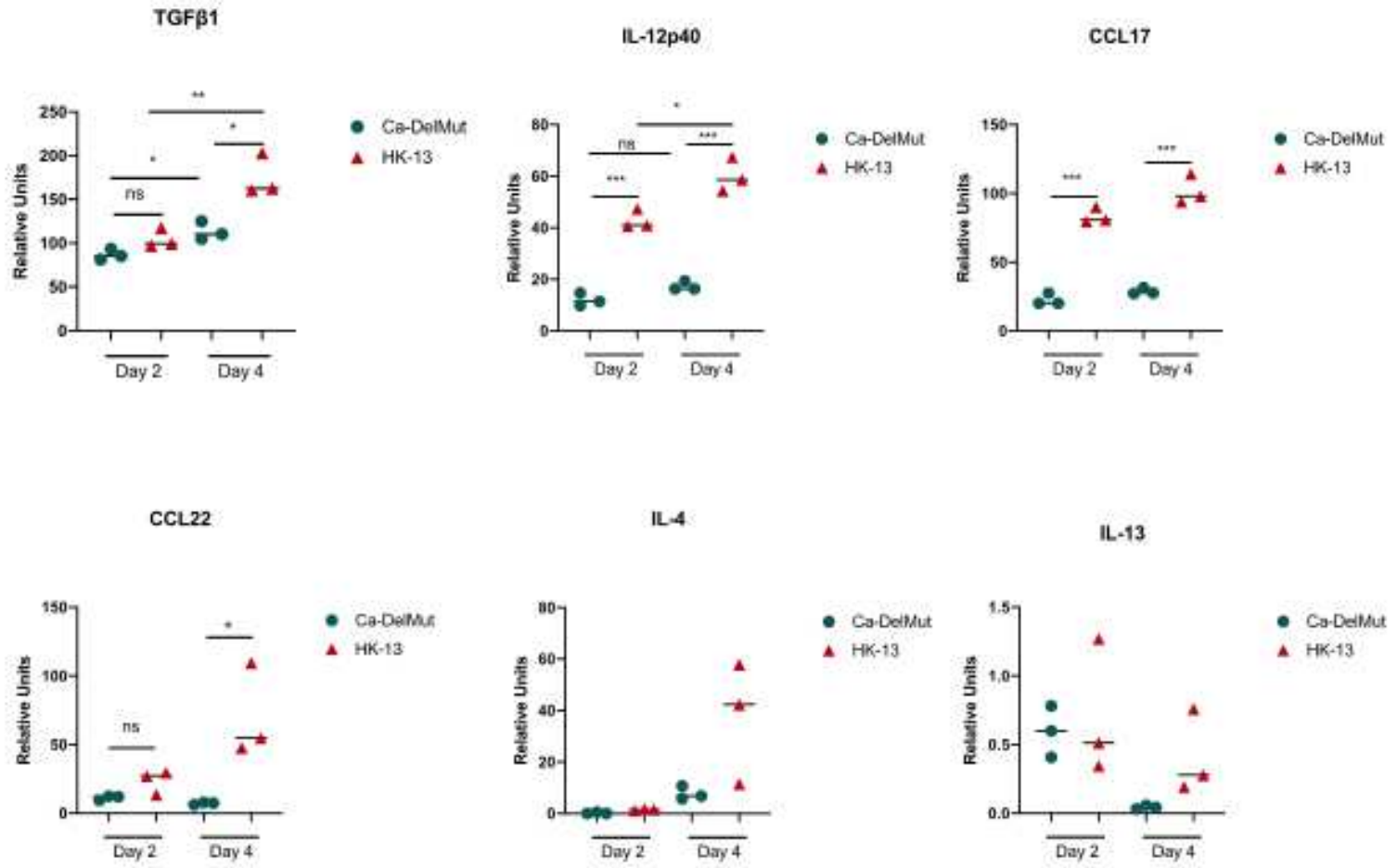

Figure S3. Ca-DelMut does not induce elevated levels of proinflammatory cytokines in hamsters. Hamsters were infected intranasally with $1 \times 10^{3}$ pfu of either Ca-DelMut or WT HK13 virus. At days 2 and 4, RNA was extracted from lung and nasal turbinate tissues using RNAsol RT procedures. cDNA was synthesized using oligo dT primers. Expression of different proinflammatory cytokines was examined by qPCR, normalized to the internal reference gene (hamster $\gamma$-actin), and the comparative $\mathrm{Ct}(2-\Delta \Delta \mathrm{Ct})$ method was utilized to calculate the cytokine expression profile. Statistical comparisons between means were performed by Student's t-test: ${ }^{* * *} \mathrm{p}<0.001,{ }^{* *} \mathrm{p}<0.01,{ }^{*} \mathrm{p}<0.05$, ns: not significant. 
Figure S4

$\mathbf{a}$

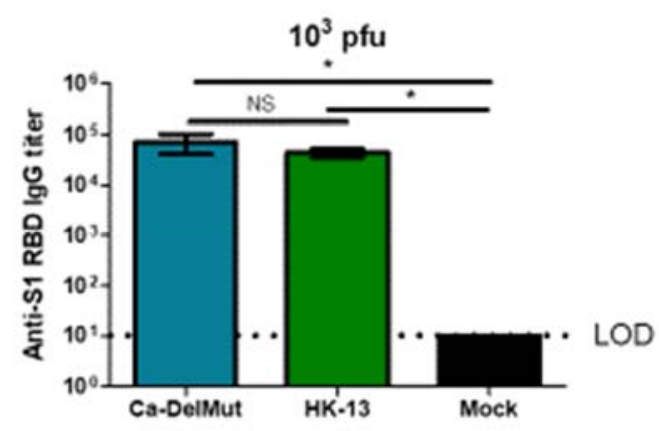

b

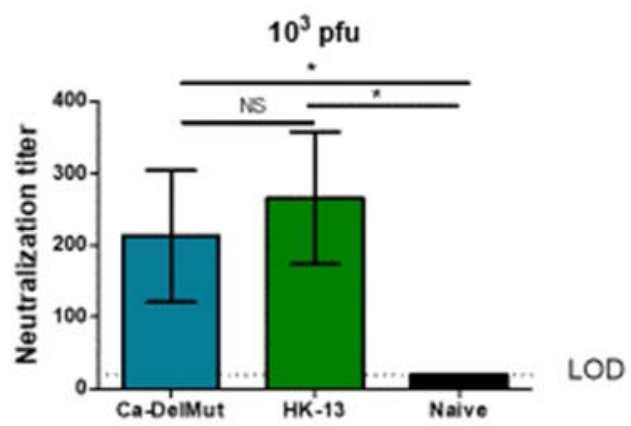

Figure S4. Lower dose $\left(10^{3} \mathrm{pfu}\right) \mathrm{Ca}-\mathrm{DelMut}$ immunization is able to induce a strong humoral response in hamsters. Hamsters were infected intranasally with $1 \times 10^{3}$ pfu of either Ca-DelMut or HK-13 virus, or mock immunized. At day 21, blood was collected from hamsters and tested for anti-S1 RBD IgG titers (a) and neutralization activity against HK-13 virus (b). Error bars represent mean \pm s.d. $(n=3)$. LOD: level of detection. 
Figure S5
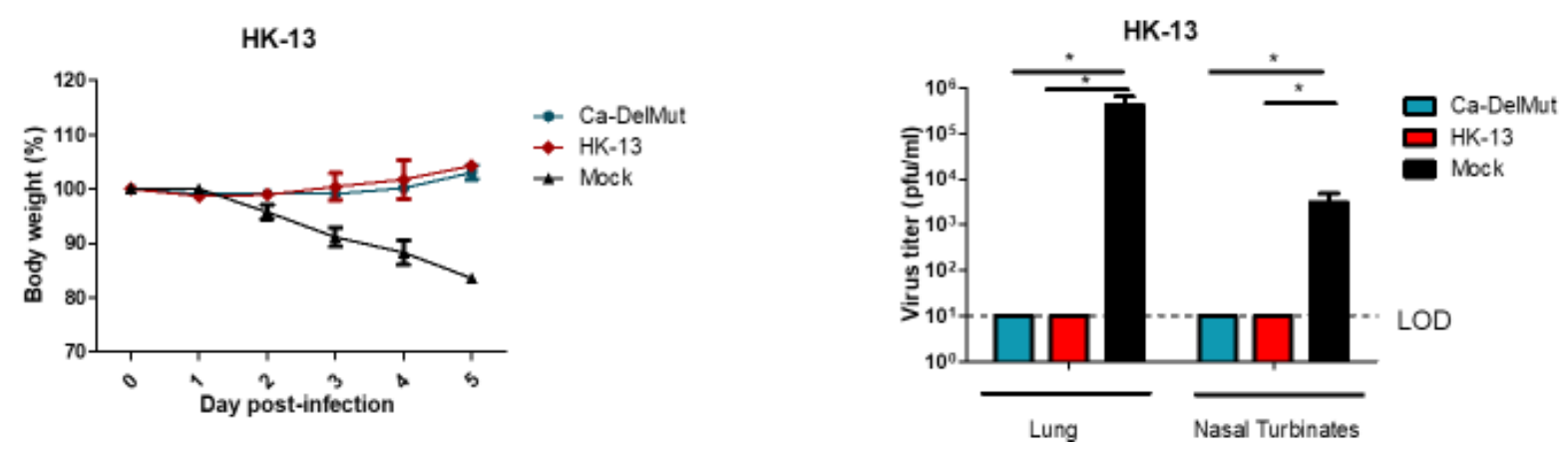

Figure S5. Lower dose Ca-DelMut immunization still provides complete protection against WT HK-13 virus. Hamsters were infected intranasally with $1 \times 10^{3}$ pfu of either Ca-DelMut or HK-13 virus, or mock immunized. At day 28 after immunization, hamsters were challenged with $1 \times 10^{3}$ pfu of HK-13 virus. Body weight change and disease symptoms were monitored for 5 days. At day 5 post-infection, lungs and nasal turbinate tissues were collected for virus titration. Error bars represent mean \pm s.d. $(n=3)$. 


\section{Figure S6}

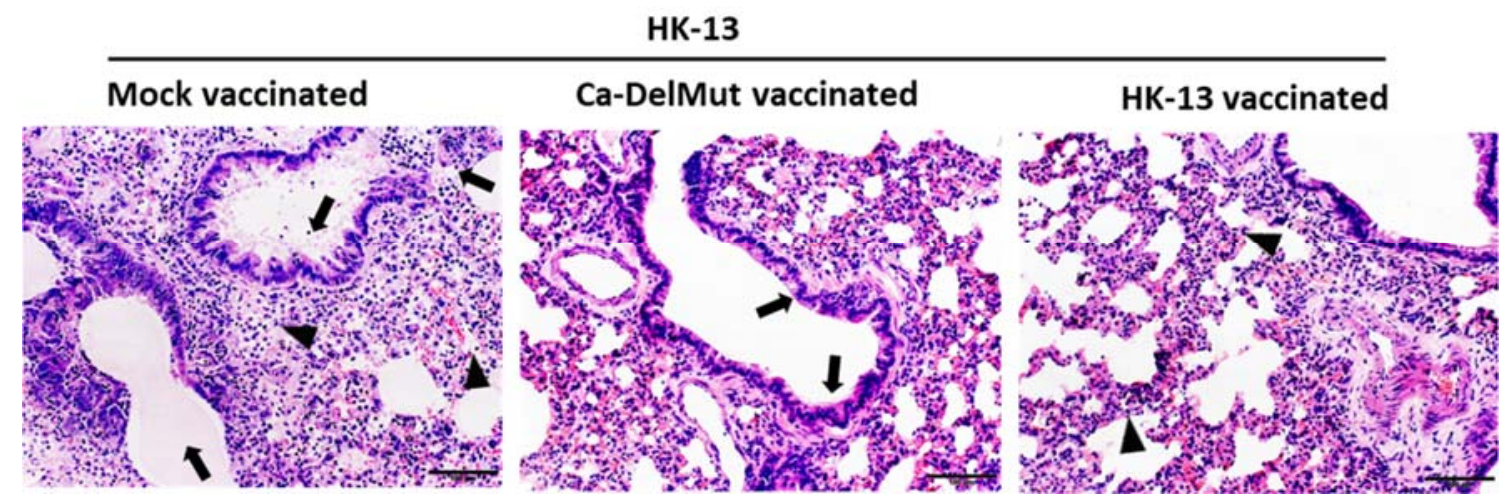

Figure S6. Low inoculum of Ca-DelMut provides protection againstreinfection of wild type SARS-CoV-2. Hamsters were infected intranasally with $1 \times 10^{3}$ pfu of either Ca-DelMut, HK13 strain or mock immunized. At day 28 after immunization, hamsters were challenged with $1 \times 10^{3}$ pfu of HK-13 virus. At day 5 post infection, lungs were collected for histopathological study. Lungs were fixed in 10\% formalin, and then processed in paraffin blocks and H\&E staining. Mock vaccinated hamster lung showed bronchiolar epithelial cells death and luminal secretion mixed with cell debris (arrows); diffuse alveolar infiltration and exudation (arrowheads); Ca-DelMut immunized lung showed no apparent bronchiolar epithelium cell death (arrows), alveoli showed regional septal infiltrationIn HK-13 strain immunized hamster lung showed no apparent histopathology other than alveolar wall thickening (arrowheads) after re-challenge. 


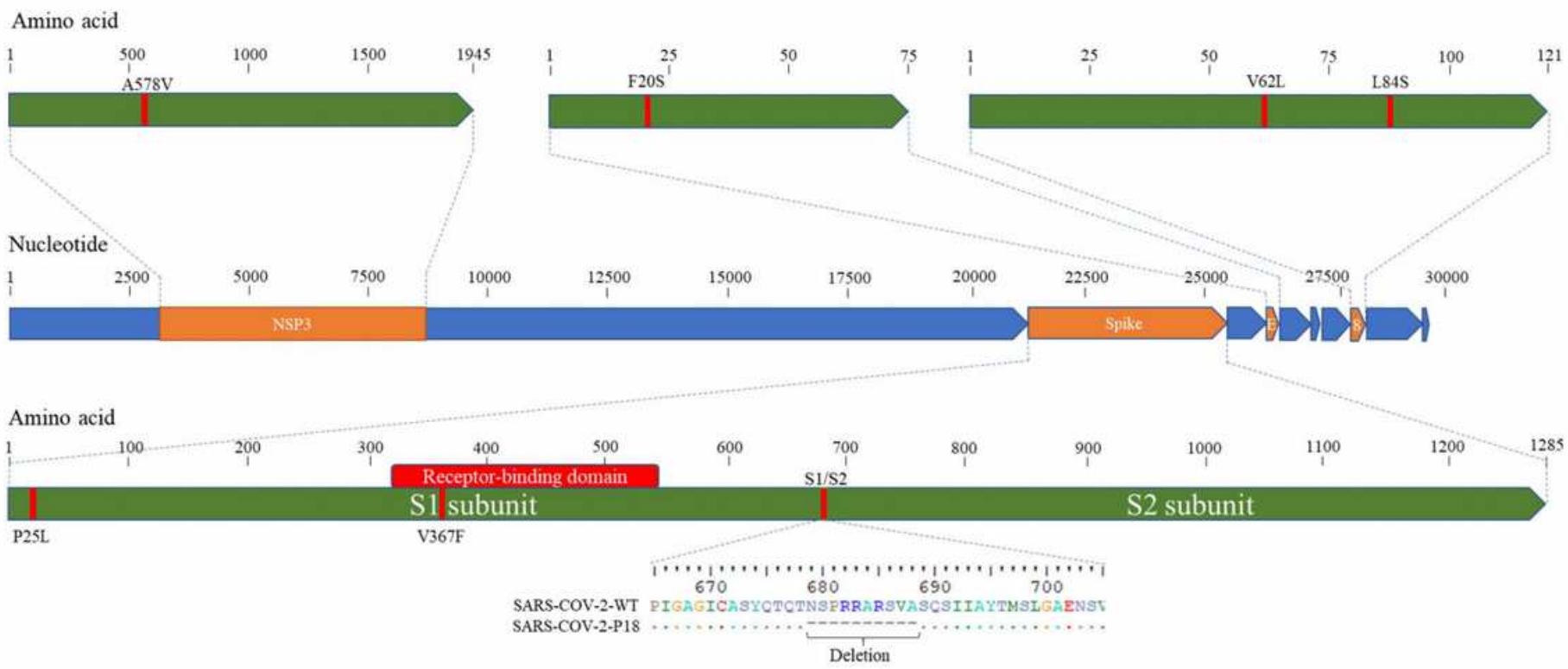

Figure 1

Schematic diagram of the SARS-CoV-2 genome showing the deletion and mutations of Ca-DelMut. DelMut- 1 virus 15 was serially passaged in Vero E6 cells at 33oC (10 passages) and 30oC (8 passages). Virus from the 18th passage was designated as Ca-DelMut live attenuated SARS-CoV-2 virus and amplified to prepare a virus stock. Ca-DelMut was sequenced by the Sanger method; mutations are shown in the diagram and in Supplementary Table 1.

a

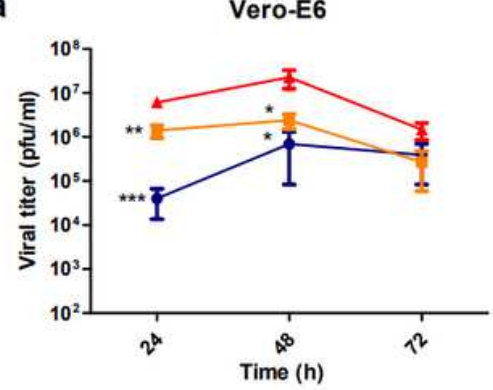

b

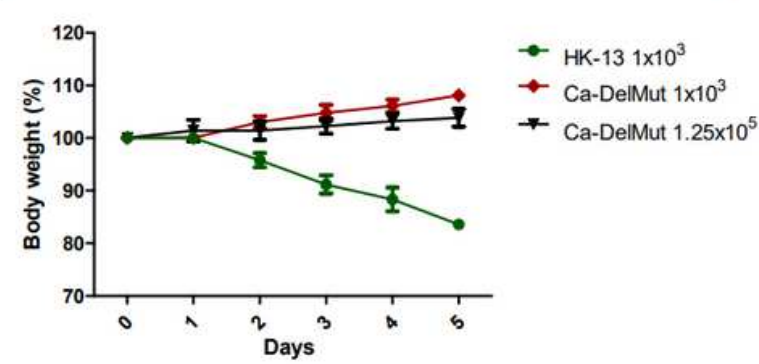

$\rightarrow$ HK-13

$\rightarrow$ Del-Mut-1

- Ca-DelMut
Calu-3

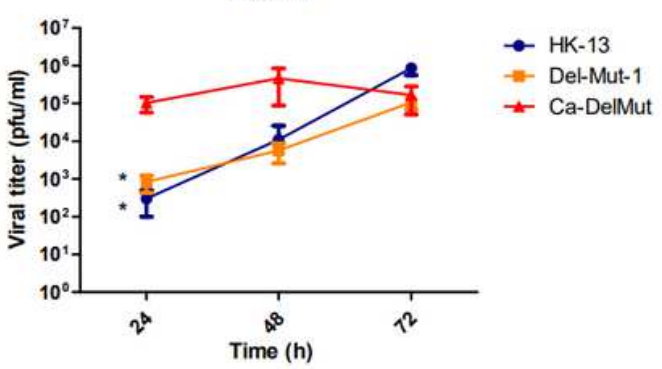

C

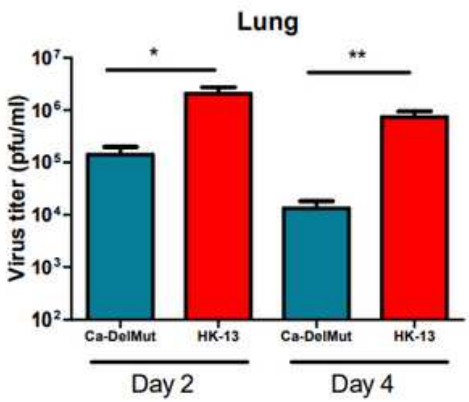

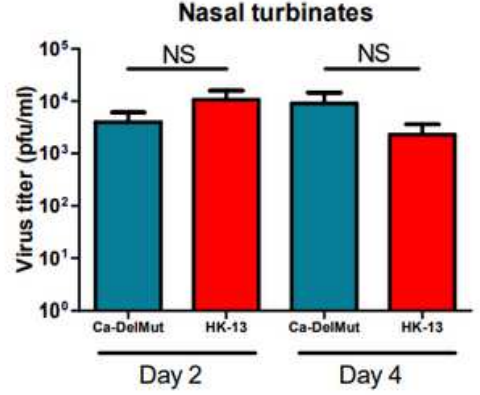


Figure 2

Replication efficiency of Ca-DelMut in vitro and in vivo. (A) Vero E6 or Calu-3 cells were infected with CaDelMut and other viruses at $0.01 \mathrm{moi}$ and cultured at $37 \mathrm{oC}$. At the indicated time points, supernatants were collected, and virus titer determined by plaque assay in Vero E6 cells. (B) Ca-DelMut infection in hamsters. Hamsters were infected intranasally with either Ca-DelMut or wild type (WT) viruses at different doses, as indicated. Body weight was monitored for 5 days. (C) Replication of Ca-DelMut in lung and nasal turbinate tissues. After virus challenge (1×103 pfu), lung and nasal turbinate tissues were collected from hamsters at days 2 and 4, then homogenized and virus titer determined. Error bars represent mean \pm s.d. $(n=3)$. Statistical comparisons between means were performed by Student's t-test: ${ }^{\star \star *} p<0.001$, $* \star$ $p<0.01,{ }^{*} p<0.05$, NS: not significant.
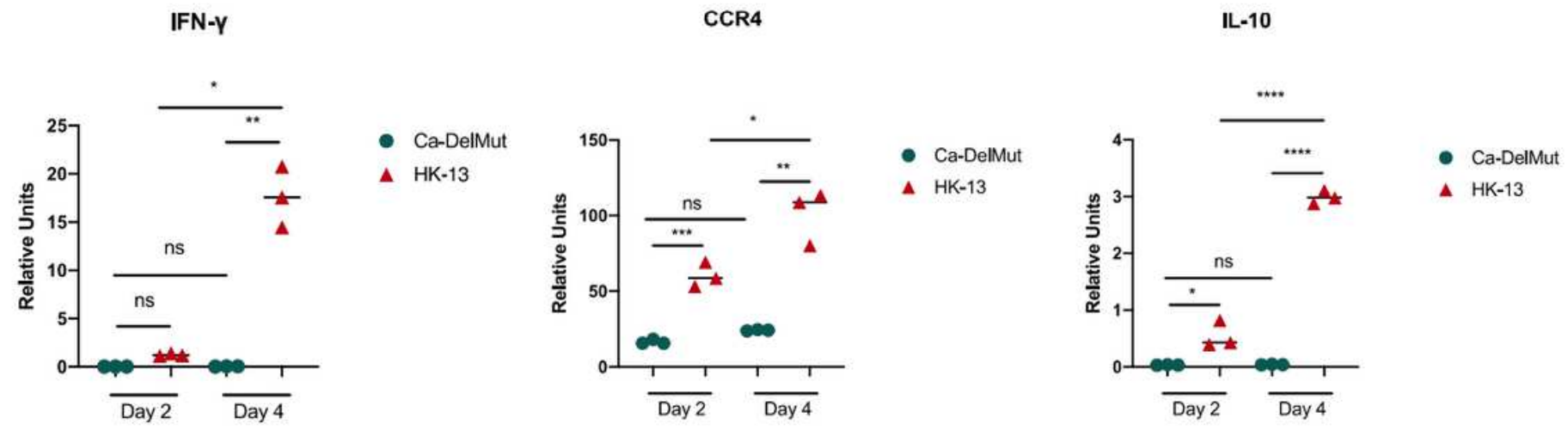

IL-21

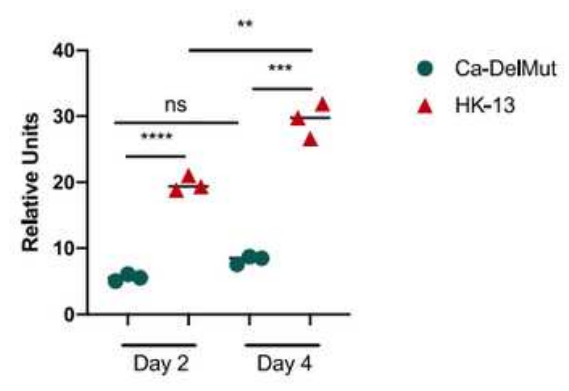

TNF- $\alpha$

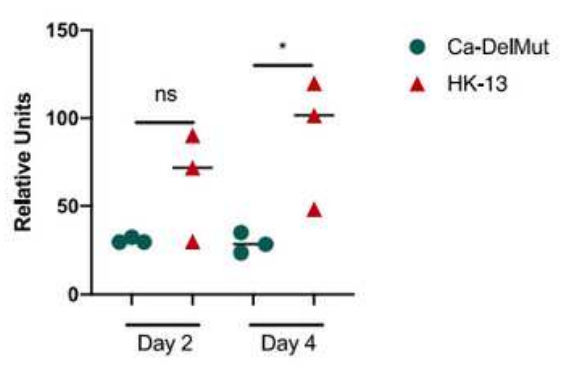

IL-6

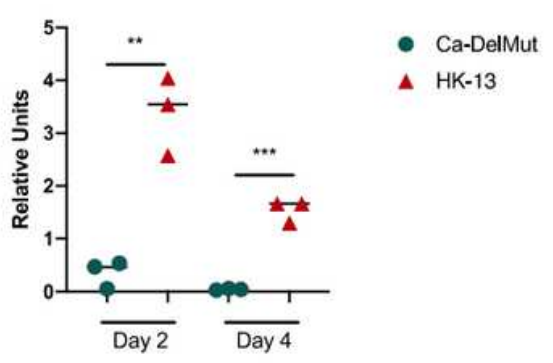

\section{Figure 3}

Proinflammatory cytokine response profiles in Ca-DelMut and wild type virus infected hamsters. Hamsters were infected intranasally with $1 \times 103$ pfu of either Ca-DelMut or WT HK-13 virus. At days 2 and 4, RNA was extracted from lung tissues of infected hamsters and cDNA synthesized using oligo dT primers. Expression of different proinflammatory cytokines was examined by qPCR, normalized to an internal reference gene (hamster $\gamma$-actin), and the comparative $\mathrm{Ct}(2-\Delta \Delta \mathrm{Ct}$ ) method utilized to calculate the cytokine expression profile. Statistical comparisons between means were performed by Student's ttest: ${ }^{\star * \star *} p<0.0001,{ }^{* \star *} p<0.001,{ }^{* \star} p<0.01,{ }^{\star} p<0.05$, ns: not significant. 
a

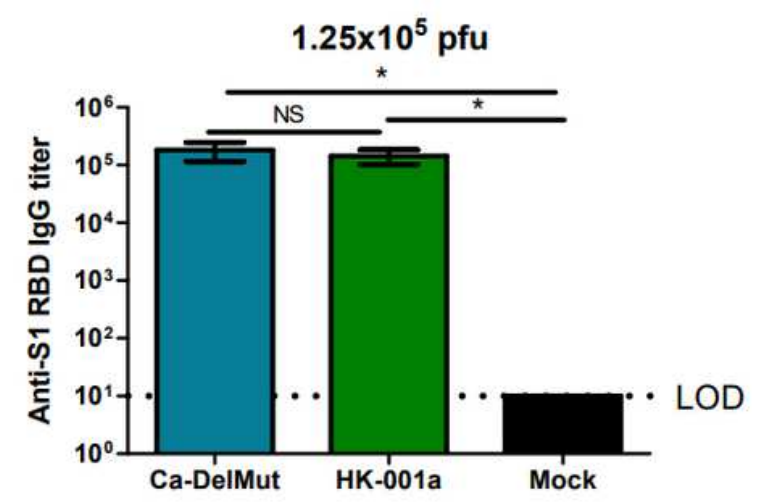

b

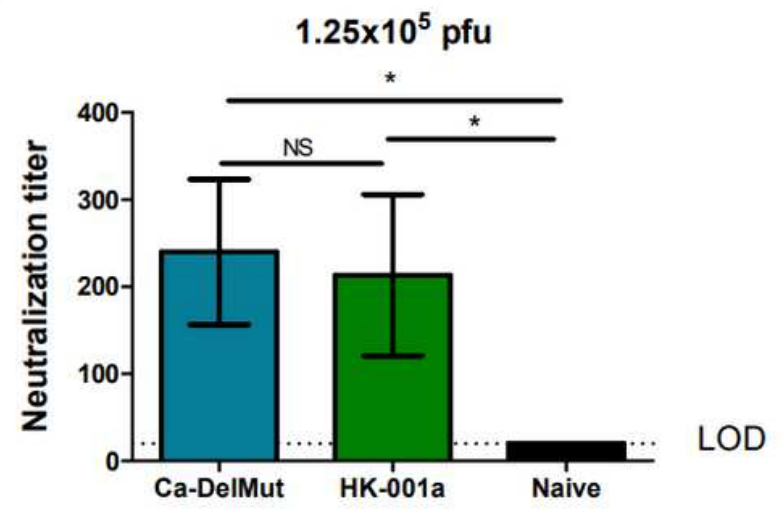

Figure 4

Antibodies induced by Ca-DelMut immunization of hamsters. Hamsters were immunized intranasally with $1.25 \times 105$ pfu of either Ca-DelMut or wild type virus (HK-001a) virus15,22, or mock immunized. At day 21, blood was collected from hamsters and tested for (A) anti-S1 RBD specific IgG titers and (B) neutralization activity against the HK-13 virus strain. Error bars represent mean $\pm s . d$. $(n=3)$. LOD: level of detection. Statistical comparisons between means were performed by Student's t-test: * $p<0.05$, ns: not significant

A

HK-13

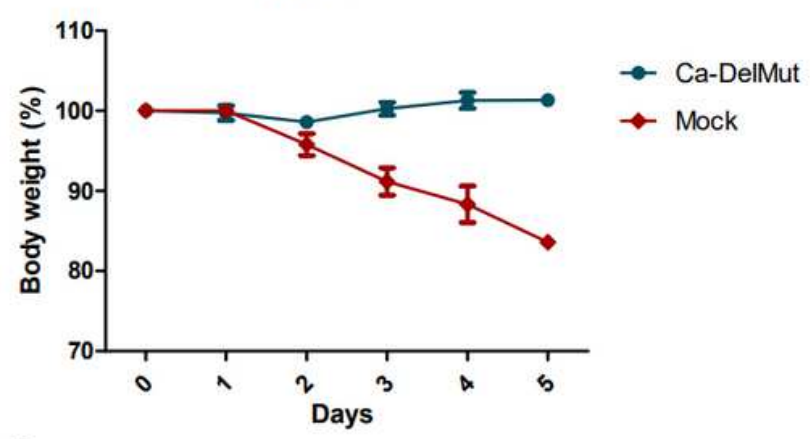

B

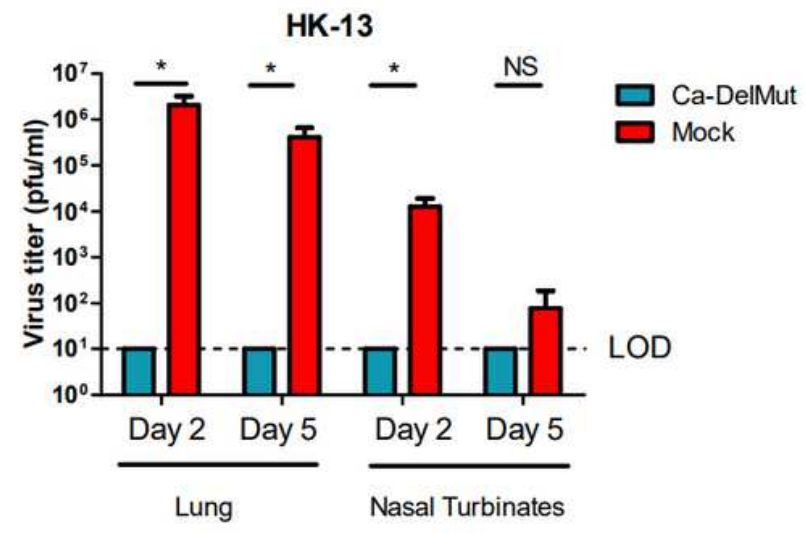

HK-95

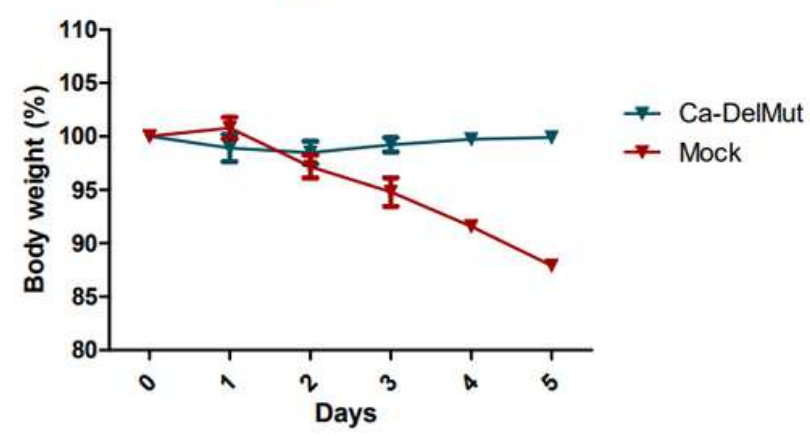

HK-95

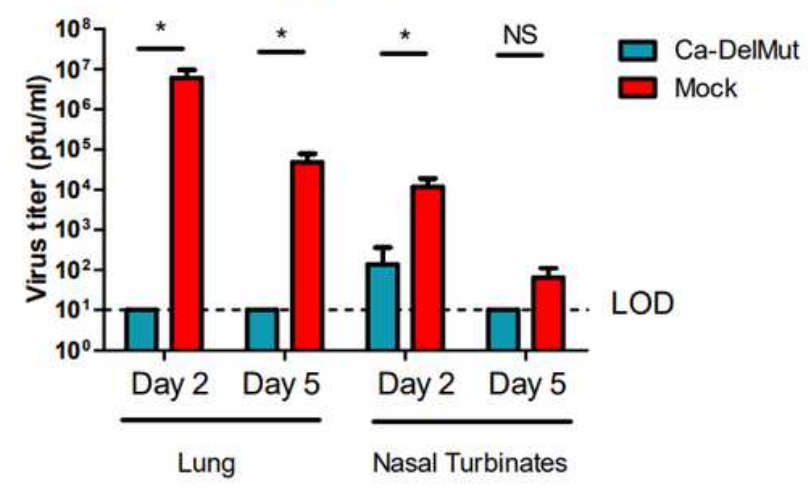

Figure 5 
Ca-DelMut immunization protection against WT virus challenge in hamsters. Hamsters were inoculated with 1.25x105 pfu Ca-DelMut or mock immunized. At day 28 after immunization, hamsters were challenged with $1 \times 103$ pfu of either HK-13 or HK-95 virus. (A) Body weight and disease symptoms were monitored for 5 days. (B) At days 2 and 5 post- infection, lungs and nasal turbinate tissues were collected for virus titration and histopathological study. Error bars represent mean \pm s.d. $(n=3)$.
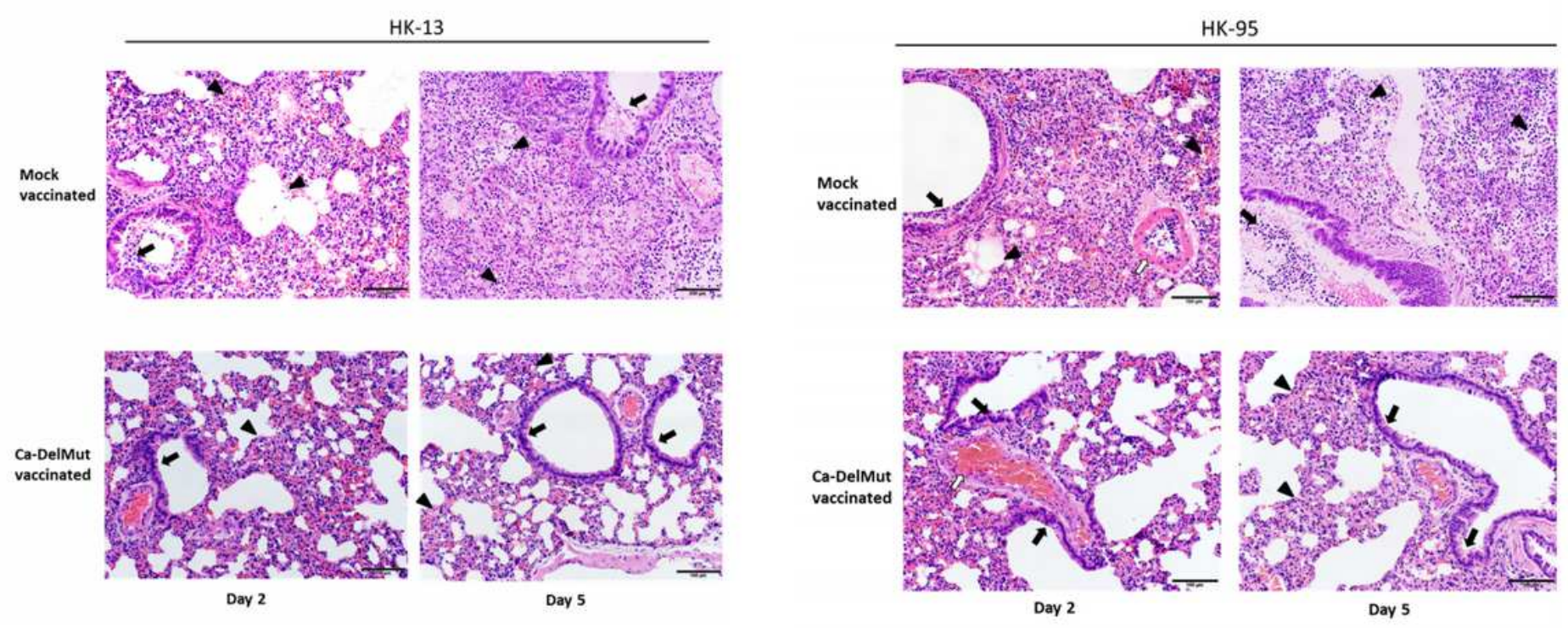

\section{Figure 6}

Histopathological analysis of lung pathology in WT virus challenged Ca- DelMut- and mock-immunized hamsters. At day 28 after immunization, hamsters were challenged with $1 \times 103$ pfu of either HK-13 or HK95 virus. At days 2 and 5 post-infection, lungs were collected, fixed, processed into paraffin blocks and sections H\&E stained. (a) Challenge with HK-13. Day 2: Mock-vaccinated hamster lungs showed bronchiolar epithelial cell death and the bronchiolar lumen filled with exudate and cell debris (arrow). Diffuse alveolar infiltration and focal hemorrhage were also seen (arrowheads). Ca-DelMut- vaccinated hamster lungs showed regional alveolar septal infiltration and blood vessel congestion (arrowhead), but no obvious bronchiolar epithelial cell death (arrow). Day 5: Lungs of mock-vaccinated hamsters showed severe alveolar infiltration and exudation (arrowheads), as well as bronchiolar luminal exudation (arrow). Vaccinated hamster lungs showed focal alveolar septal infiltration (arrowheads), while the bronchiolar epithelium appeared normal with no luminal secretion or cell debris (arrows).

\section{Supplementary Files}

This is a list of supplementary files associated with this preprint. Click to download.

- Supplementarylnformation.pdf 